\title{
Optimal prefix codes for pairs of geometrically-distributed random variables
}

\author{
Frédérique Bassino, Julien Clément, Gadiel Seroussi, Fellow, IEEE, and Alfredo Viola
}

\begin{abstract}
Optimal prefix codes are studied for pairs of independent, integer-valued symbols emitted by a source with a geometric probability distribution of parameter $q, 0<q<1$. By encoding pairs of symbols, it may be possible to reduce the redundancy penalty of symbol-by-symbol encoding, while preserving the simplicity of the encoding and decoding procedures typical of Golomb codes and their variants. It is shown that optimal codes for these so-called two-dimensional geometric distributions are parameter-singular, in the sense that a prefix code that is optimal for one value of the parameter $q$ cannot be optimal for any other value of $q$. This is in sharp contrast to the one-dimensional case, where codes are optimal for positive-length intervals of the parameter $q$. Thus, in the two-dimensional case, it is infeasible to give a compact characterization of optimal codes for all values of the parameter $q$, as was done in the one-dimensional case. Instead, optimal codes are characterized for a discrete sequence of values of $q$ that provides good coverage of the unit interval. Specifically, optimal prefix codes are described for $q=2^{-1 / k}$ ( $k \geq 1)$, covering the range $q \geq \frac{1}{2}$, and $q=2^{-k}(k>1)$, covering the range $q<\frac{1}{2}$. The described codes produce the expected reduction in redundancy with respect to the one-dimensional case, while maintaining low complexity coding operations.
\end{abstract}

Index terms-geometric distributions, prefix codes, Huffman codes, Golomb codes, codes for countable alphabets, lossless compression

\section{INTRODUCTION}

In 1966, Golomb [1] described optimal binary prefix codes for some geometric distributions over the nonnegative integers, namely, distributions with probabilities $p(i)$ of the form

$$
p(i)=(1-q) q^{i}, \quad i \geq 0,
$$

for some real-valued parameter $q, 0<q<1$. In [2], these Golomb codes were shown to be optimal for all geometric distributions. These distributions occur, for example, when

This work was supported in part by ECOS project U08E02, by PDT project 54/178 2006-2008, and by CSIC project (Universidad de la República) fondos 2009-2011. A. Viola's work was done in part while he was visiting GREYC, Université de Caen and the Laboratoire d'Informatique GaspardMonge, Université de Marne la Vallée, France. Parts of this paper were presented at the 2006 Data Compression Conference, and at the 2006 IEEE International Symposium on Information Theory.

F. Bassino is with LIPN UMR 7030. Université Paris 13 - CNRS, France (e-mail: bassino@lipn.univ-paris13.fr).

J. Clément is with GREYC UMR 6072, CNRS, Université de Caen, ENSICAEN, France (e-mail: julien.clement@unicaen.fr).

G. Seroussi is with Hewlett-Packard Laboratories, Palo Alto, CA 94304 USA, and with Facultad de Ingeniería, Universidad de la República, Montevideo, Uruguay (e-mail:gseroussi@ieee.org).

A. Viola is with Instituto de Computación, Facultad de Ingeniería, Universidad de la República, Montevideo, Uruguay (e-mail: viola@fing.edu.uy).

Copyright (c) 2012 IEEE. Personal use of this material is permitted. However, permission to use this material for any other purposes must be obtained from the IEEE by sending a request to pubs-permissions @ ieee.org. encoding run lengths (the original motivation in [1]), and in image compression when encoding prediction residuals, which are well-modeled by two-sided geometric distributions. Optimal codes for the latter were characterized in [3], based on some combinations and variants of Golomb codes. Codes based on the Golomb construction have the practical advantage of allowing the encoding of a symbol $i$ using a simple explicit computation on the integer value of $i$, without recourse to nontrivial data structures or tables. This has led to their adoption in many practical applications (cf. [4],[5]).

Symbol-by-symbol encoding, however, can incur significant redundancy relative to the entropy of the distribution, even when dealing with sequences of independent, identically distributed random variables. One way to mitigate this problem, while keeping the simplicity and low latency of the encoding and decoding operations, is to consider short blocks of $d>1$ symbols, and use a prefix code for the blocks. In this paper, we study optimal prefix codes for pairs (blocks of length $d=2$ ) of independent, identically distributed geometric random variables, namely, distributions on pairs of nonnegative integers $(i, j)$ with probabilities of the form

$$
P(i, j)=p(i) p(j)=(1-q)^{2} q^{i+j} \quad i, j \geq 0 .
$$

We refer to this distribution as a two-dimensional geometric distribution (TDGD), defined on the alphabet of integer pairs $\mathcal{A}=\{(i, j) \mid i, j \geq 0\}$. For succinctness, we denote a TDGD of parameter $q$ by $\operatorname{TDGD}(q)$.

Aside from the mentioned practical motivation, the problem is of intrinsic combinatorial interest. It was proved in [6] (see also [7]) that, if the entropy $-\sum_{a \in \mathcal{A}} P(a) \log P(a)$ of a distribution over a countable alphabet $\mathcal{A}$ is finite, optimal codes exist and can be obtained, in the limit, from Huffman codes for truncated versions of the alphabet. However, the proof does not give a general way for effectively constructing optimal codes, and in fact, there are few families of distributions over countable alphabets for which an effective construction is known [8][9]. An algorithmic approach to building optimal codes is presented in [9], which covers geometric distributions and various generalizations. The approach, though, is not applicable to TDGDs, as explicitly noted in [9].

Some characteristic properties of the families of optimal codes for geometric and related distributions in the onedimensional case turn out not to hold in the two-dimensional case. Specifically, the optimal codes described in [1] and [3] correspond to binary trees of bounded width, namely, the

\footnotetext{
${ }^{1} \log x$ and $\ln x$ will denote, respectively, the base- 2 and the natural
} logarithm of $x$. 
number of codewords of any given length is upper-bounded by a quantity that depends only on the code parameters. Also, the family of optimal codes in each case partitions the parameter space into regions of positive volume, such that all the corresponding distributions in a region admit the same optimal code. These properties do not hold in the case of optimal codes for TDGDs. In particular, optimal codes for TDGDs turn out to be parameter-singular, in the sense that if a code $\mathcal{T}_{q}$ is optimal for $\operatorname{TDGD}(q)$, then $\mathcal{T}_{q}$ is not optimal for $\operatorname{TDGD}\left(q^{\prime}\right)$ for any parameter value $q^{\prime} \neq q$. This result is presented in Section III. (A related but somewhat dual problem, namely, counting the number of distinct trees that can be optimal for a given source over a countable alphabet, is studied in [10].)

An important consequence of this singularity is that any set containing optimal codes for all values of $q$ must be uncountable, and, thus, it would be infeasible to give a compact characterization of such a set, as was done in [1] or [3] for one-dimensional cases ${ }^{2}$ Thus, from a practical point of view, the best we can expect is to characterize optimal codes for countable sequences of parameter values. In this paper, we present such a characterization, for a sequence of parameter values that provides good coverage of the range of $0<q<1$. Specifically, in Section IV] we describe the construction of optimal codes for $\operatorname{TDGD}(q)$ with $q=2^{-1 / k}$ for integers $k \geq 1$. covering the range $q \geq \frac{1}{2}$, and in Section $\mathrm{V}$, we do so for $\operatorname{TDGD}(q)$ with $q=2^{-k}$ for integers $k>1$, covering the range $q<\frac{1}{2}$ (thus, overall, we show optimal codes for all values of $q$ such that $-\log q$ is either an integer or the inverse of one). In the case $q<\frac{1}{2}$, we observe that, as $k \rightarrow \infty$ $(q \rightarrow 0)$, the optimal codes described converge to a limit code, in the sense that the codeword for any given pair $(a, b)$ remains the same for all $k>k_{0}(a, b)$, where $k_{0}$ is a threshold that can be computed from $a$ and $b$ (this limit code is also mentioned, without proofs, in [11]). The codes in both constructions are of unbounded width. However, they are regular [12], in the sense that the corresponding infinite trees have only a finite number of non-isomorphic whole subtrees (i.e., subtrees consisting of a node and all of its descendants). This allows for deriving recursions and explicit expressions for the average code length, as well as feasible encoding/decoding procedures. Notice that, to the best of our knowledge, the only case for which an optimal code for a TDGD had been characterized prior to this work was the trivial case $q=\frac{1}{2}$, in which case encoding each component of $(i, j)$ separately with a unary code (i.e., a Golomb code of order one) has zero redundancy, and is thus optimal (cf. also [11]).

Practical considerations, and the redundancy of the new codes, are discussed in Section VI. where we present redundancy plots and comparisons with symbol-by-symbol Golomb coding and with the optimal code for a TDGD for each plotted value of $q$ (optimal average code lengths for arbitrary values of $q$ were estimated numerically to sufficiently high preci-

\footnotetext{
${ }^{2}$ Loosely, by a compact characterization we mean one in which each code is characterized by a finite number of finite parameters, which drive the corresponding encoding/decoding procedures.

${ }^{3}$ These are the same distributions for which optimality of Golomb codes was originally established in [1].
}

sion). We also derive an exact expression for the asymptotic oscillatory behavior of the redundancy of the new codes as $q \rightarrow 1$. The study confirms the redundancy gains over symbolby-symbol encoding with Golomb codes, and the fact that the discrete sequence of codes presented provides a good approximation to the full class of optimal codes over the range of the parameter $q$.

Our constructions and proofs of optimality rely on the technique of Gallager and Van Voorhis [2], which was also used in [3]. As noted in [2], most of the work and ingenuity in applying the technique goes into discovering appropriate "guesses" of the basic components on which the construction iterates, and in describing the structure of the resulting codes. With the correct guesses, the proofs are straightforward. The technique of [2] is reviewed in Section [II] where we also introduce some definitions and notation that will be useful throughout the paper.

\section{Preliminaries}

\section{A. Definitions}

We are interested in encoding the alphabet $\mathcal{A}$ of integer pairs $(i, j), i, j \geq 0$, using a binary prefix code $C$ (we will refer to $C$ plainly as a code, the binary and prefix properties assumed throughout). As usual, we associate $C$ with a rooted (infinite) binary tree, whose leaves correspond, bijectively, to symbols in $\mathcal{A}$, and where each branch is labeled with a binary digit. The binary codeword assigned to a symbol is "read off" the labels on the path from the root to the corresponding leaf. The depth of a node $x$ in a tree $T$, denoted $\operatorname{depth}_{T}(x)$, is the number of branches on the path from the root to $x$. By extension, the depth (or height) of a finite tree is defined as the maximal depth of any of its nodes. A level of $T$ is the set of all nodes at a given depth $\ell$ (we refer to this set as level $\ell$ ). Let $n_{\ell}^{T}$ denote the number of leaves in level $\ell$ of $T$ (we will sometimes omit the superscript $T$ when clear from the context). We refer to the sequence $\left\{n_{\ell}^{T}\right\}_{\ell \geq 0}$ as the profile of $T$. Two trees will be considered equivalent if their profiles are identical. Thus, for a code $C$, we are only interested in its tree profile, or, equivalently, the length distribution of its codewords. Given the profile of a tree, and an ordering of $\mathcal{A}$ in decreasing probability order, it is always possible to define a canonical tree (say, by assigning leaves in alphabetical order; see, e.g., [13]) that uniquely defines a code for $\mathcal{A}$. The notion of tree equivalence adopted implies that given a tree, we can arbitrarily permute the nodes at any level, since such a permutation leaves the profile invariant. This will allow us to make, without loss of generality, certain assumptions on the structure of the tree. In particular, we will often make the assumption that if a tree contains, say, at least $2^{j}$ leaves at a certain level $\ell$, then there is a set of $2^{j}$ leaves at level $\ell$ that have a common ancestor ${ }^{4} \nu$ at level $\ell-j$ (an alphabetically ordered tree, in fact, always has this property).

With a slight abuse of terminology, we will not distinguish between a code and its corresponding tree (or profile), and will

\footnotetext{
${ }^{4}$ We use the usual "family" terminology for trees: nodes have children, parents, ancestors and descendants. We also use the common convention of visualizing trees with the root at the top and leaves at the bottom. Thus, ancestors are "up," and descendants are "down."
} 
refer to the same object sometimes as a tree and sometimes as a code. Unless noted otherwise, all trees considered in this paper are full, i.e., every node in the tree is either a leaf or the parent of two children (full trees are sometimes referred to in the literature as complete). A tree is balanced (or uniform) if it has $2^{k}$ leaves, all of them at depth $k$, for some $k \geq 0$. We denote such a tree by $\mathcal{U}_{k}$. We will restrict the use of the term subtree to refer to whole subtrees of $T$, i.e., subtrees that consist of a node and all of its descendants in $T$.

We call $s(i, j)=i+j$ the signature of $(i, j) \in \mathcal{A}$. For a given value $s=s(i, j)$, there are $s+1$ pairs with signature $s$, all with the same probability, $P(s)=(1-q)^{2} q^{s}$, under the distribution (1). Given a code $C$, symbols of the same signature can be freely permuted without affecting the properties of interest to us (e.g., average code length). Thus, for simplicity, we can also regard the correspondence between leaves and symbols as one between leaves and elements of the multiset

$$
\hat{\mathcal{A}}=\{0,1,1,2,2,2, \ldots, \underbrace{s, \ldots, s}_{s+1 \text { times }}, \ldots\} .
$$

In constructing the tree, we do not distinguish between different occurrences of a signature $s$; for actual encoding, the $s+1$ leaves labeled with $s$ are mapped to the symbols $(0, s),(1, s-1), \ldots,(s, 0)$ in some fixed order. In the sequel, we will often ignore normalization factors for the signature probabilities $P(s)$ (in cases where normalization is inconsequential), and will use instead weights $w(s)=q^{s}$.

Consider a tree (or code) $T$ for $\mathcal{A}$. Let $U$ be a subtree of $T$, and let $s(x)$ denote the signature associated with a leaf $x$ of $U$. Let $F(U)$ denote the set of leaves of $U$, referred to as its fringe. We define the weight, $w_{q}(U)$, of $U$ as

$$
w_{q}(U)=\sum_{x \in F(U)} q^{s(x)},
$$

and the cost, $\mathcal{L}_{q}(U)$, of $U$ as

$$
\mathcal{L}_{q}(U)=\sum_{x \in F(U)} \operatorname{depth}_{U}(x) q^{s(x)}
$$

(the subscript $q$ may be omitted when clear from the context). When $U=T$, we have $w_{q}(T)=(1-q)^{-2}$, and $\overline{\mathcal{L}}_{q}(T) \triangleq$ $(1-q)^{2} \mathcal{L}_{q}(T)$ is the average code length of $T$. A tree $T$ is optimal for $\operatorname{TDGD}(q)$ if $\mathcal{L}_{q}(T) \leq \mathcal{L}_{q}\left(T^{\prime}\right)$ for any tree $T^{\prime}$.

\section{B. Some basic objects and operations}

For $\alpha \geq 1$, we say that a finite source with probabilities $p_{1} \geq p_{2} \geq \cdots \geq p_{N}, N \geq 2$, is $\alpha$-uniform if $p_{1} / p_{N} \leq \alpha$. A 2-uniform source is also called quasi-uniform. An optimal code for a quasi-uniform source on $N$ symbols consists of $2^{\lceil\log N\rceil}-N$ codewords of length $\lfloor\log N\rfloor$, and $2 N-2^{\lceil\log N\rceil}$ codewords of length $\lceil\log N\rceil$, the shorter codewords corresponding to the more probable symbols [2]. We refer to such a code (or the associated tree) also as quasi-uniform, denote it by $Q_{N}$, and denote by $Q_{N}(i)$ the codeword it assigns to the symbol associated with $p_{i}, 1 \leq i \leq N$. For convenience, we define $Q_{1}$ as a null code, which assigns code length zero to the single symbol in the alphabet. Clearly, for integers $k \geq 0$, we have $Q_{2^{k}}=\mathcal{U}_{k}$. The fringe thickness of a finite tree $T$, denoted $f_{T}$, is the maximum difference between the depths of any two leaves of $T$. Quasi-uniform trees $T$ have $f_{T} \leq 1$, while uniform trees have $f_{T}=0$. In Section IV we present a characterization of optimal codes of fringe thickness two for 4-uniform distributions, which generalizes the quasi-uniform case. This generalization will help in the characterization of the optimal codes for $\operatorname{TDGD}(q), q=2^{-1 / k}$.

The concatenation of two trees $T$ and $U$, denoted $T \cdot U$, is obtained by attaching a copy of $U$ to each leaf of $T$. Regarded as a code, $T \cdot U$ consists of all the possible concatenations $t \cdot u$ of a word $t \in T$ with one $u \in U$. The Golomb code of order $k \geq 1$ [1], denoted $G_{k}$, encodes an integer $i$ by concatenating $Q_{k}(i \bmod k)$ with a unary encoding of $\lfloor i / k\rfloor$ (e.g., $\lfloor i / k\rfloor$ ones followed by a zero). The first-order Golomb code $G_{1}$ is just the unary code, whose corresponding tree consists of a root with one leaf child on the branch labeled '0', and, recursively, a copy of $G_{1}$ attached to the child on the branch labeled '1'. Thus, we have $G_{k}=Q_{k} \cdot G_{1}$.

\section{The Gallager-Van Voorhis method}

When proving optimality of infinite codes for TDGDs, we will rely on the method due to Gallager and Van Voorhis [2], which is briefly outlined below, adapted to our setting and terminology.

- Define a sequence of finite reduced sources $\left(\mathcal{S}_{t}\right)_{t=0}^{\infty}$. The alphabet of the reduced source $\mathcal{S}_{t}$ is a multiset $\mathcal{S}_{t}=\mathcal{H}_{t} \cup \mathcal{F}_{t}$, where $\mathcal{H}_{t}$ is a multiset comprising the signatures $0,1, \ldots, s-1$ (with multiplicities as in (2)), and $\mathcal{F}_{t}$ consists of a finite number of (possibly infinite) subsets of $\hat{\mathcal{A}}$, referred to as virtual symbols, which form a partition of the remaining signatures. We naturally associate with each virtual symbol a weight equal to the sum of the weights of the signatures it contains.

- Verify that the sequence $\left(\mathcal{S}_{t}\right)_{t=0}^{\infty}$ is compatible with the bottom-up Huffman procedure. This means that after a number of merging steps of the Huffman algorithm on the reduced source $\mathcal{S}_{t}$, one gets $\mathcal{S}_{t-1}$. Proceed recursively, until $\mathcal{S}_{0}$ is obtained.

- Apply the Huffman algorithm to $\mathcal{S}_{0}$.

While the sequence of reduced sources $\mathcal{S}_{t}$ can be seen as evolving "bottom-up," the infinite code $C$ constructed results from a "top-down" sequence of corresponding finite codes $C_{t}$, whose size grows with $t$, and which unfold by recursive reversal of the mergers in the Huffman procedure. One shows that the sequence of codes $\left(C_{t}\right)_{t \geq 0}$ converges to an infinite code $C$, in the sense that for every $j \geq 1$, with codewords of $C_{t}$ consistently sorted, the $j$ th codeword of $C_{t}$ is eventually constant when $t$ grows, and equal to the $j$ th codeword of $C$. A corresponding convergence argument on the sequence of average code lengths then establishes the optimality of $C$.

This method was successfully applied to characterize infinite optimal codes in [2] and [3]. While the technique is straightforward once appropriate reduced sources are defined, the difficulty in each case is to guess the structure of these source. In a sense, this is a self-bootstrapping procedure, where one needs to guess the structure of the codes sought, and use that structure to define the reduced sources, which, in turn, 
serve to prove that the guess was correct. We will apply the Gallager-Van Voorhis method to prove optimality of codes for certain families of TDGDs in Sections IV and $\mathrm{V}$ In each case, we will emphasize the definition and structure of the reduced sources, and show that they are compatible with the Huffman procedure. We will omit the discussion on convergence, and the formal induction proofs, since the arguments are essentially the same as those in [2] and [3].

\section{PARAMETER-SINGUlARITY OF OPTIMAL CODES FOR TDGDS}

In the case of one-dimensional geometric distributions, the unit interval $(0,1)$ is partitioned into an infinite sequence of semi-open intervals $\left(q_{k-1}, q_{k}\right], k \geq 1$, such that the Golomb code $G_{k}$ is optimal for all values of the distribution parameter $q$ in $\left(q_{k-1}, q_{k}\right]$. Specifically, for $k \geq 0, q_{k}$ is the (unique) nonnegative root of the equation $q^{k}+q^{k+1}-1=0$ [2]. Thus, we have $q_{0}=0, q_{1}=(\sqrt{5}-1) / 2 \approx 0.618, q_{2} \approx 0.755$, etc. A similar property holds in the case of two-sided geometric distributions [3], where the two-dimensional parameter space is partitioned into a countable sequence of patches such that all the distributions with parameter values in a given patch admit the same optimal code. In this section, we prove that, in sharp contrast to these examples, optimal codes for TDGDs are parameter-singular, in the sense that a code that is optimal for a certain value of the parameter $q$ cannot be optimal for any other value of $q$. More formally, we present the following result.

Theorem 1: Let $q$ and $q_{1}$ be real numbers in the interval $(0,1)$, with $q \neq q_{1}$, and let $\mathcal{T}_{q}$ be an optimal tree for $\operatorname{TDGD}(q)$. Then, $\mathcal{T}_{q}$ is not optimal for $\operatorname{TDGD}\left(q_{1}\right)$.

Remark. It follows from Theorem 1 that any set containing an optimal code for each distribution $\operatorname{TDGD}(q)$, for all values of $q$, must be uncountable. This implies, in turn, that most optimal codes for TDGDs do not have finite descriptions, in sharp contrast with the one-dimensional case. From an algorithmic point of view, then, the key question is for what "interesting" countable sets of values of $q$ a full characterization of optimal codes is possible. In a theoretical sense, perhaps the ultimate such set would be that of all values of $q$ which have finite descriptions (more formally, the set of computable values of $q$ relative to some universal Turing machine; see, e.g., [14]). For this set, the goal would be to obtain a general procedure which, given a finite description of $q$, and a pair $(i, j)$, produces the corresponding codeword in an optimal code for $\operatorname{TDGD}(q)$. A somewhat less ambitious theoretical goal, although probably not less valuable from a practical point of view, would be to characterize optimal codes for a dense countable set of values of $q$, e.g., all rational values of $q$, or all values of $q$ such that $\log q$ is rational. These comprehensive characterizations appear quite challenging, and remain open problems. In Sections IV and $\mathrm{V}$ we characterize optimal codes for a "smaller" infinite countable set of TDGDs, namely, the set of distributions $\operatorname{TDGD}(q)$ such that $-\log q$ is either a positive integer or the inverse of one. It will turn out, as will be shown in Section VI, that this set provides good coverage of the interval $0<q<1$, in the sense that, given an arbitrary value $q^{\prime}$ in the interval, encoding $\operatorname{TDGD}\left(q^{\prime}\right)$ with the best available code from the characterized set results in relatively low added redundancy, and yields the expected redundancy gains over optimal symbol-by-symbol encoding with Golomb codes.

We will prove Theorem 1 through a series of lemmas, which will shed more light on the structure of optimal trees for TDGDs. For simplicity, we assume throughout that a fixed optimal tree $\mathcal{T}_{q}$ is given (for a given value of $q$ ).

Lemma 1: Leaves with a given signature $s$ are found in at most two consecutive levels of $\mathcal{T}_{q}$.

Proof: Let $d_{0}$ and $d_{1}$ denote, respectively, the minimum and maximum depths of a leaf with signature $s$ in $\mathcal{T}_{q}$. Assume, contrary to the claim of the lemma, that $d_{1}>d_{0}+1$. We transform $\mathcal{T}_{q}$ into a tree $\mathcal{T}_{q}^{\prime}$ as follows. Pick a leaf with signature $s$ at level $d_{0}$, and one at level $d_{1}$. Place both signatures $s$ as children of the leaf at level $d_{0}$, which becomes an internal node. Pick any signature $s^{\prime}$ from a level strictly deeper than $d_{1}$, and move it to the vacant leaf at level $d_{1}$. Tracking changes in the code lengths corresponding to the affected signatures, and their effect on the cost, we have

$$
\mathcal{L}_{q}\left(\mathcal{T}_{q}^{\prime}\right)=\mathcal{L}_{q}\left(\mathcal{T}_{q}\right)+q^{s}\left(d_{0}-d_{1}+2\right)-q^{s^{\prime}} \delta,
$$

where $\delta$ is a positive integer. By our assumption, the quantity multiplying $q^{s}$ in 3 is non-positive, and we have $\mathcal{L}_{q}\left(\mathcal{T}_{q}^{\prime}\right)<$ $\mathcal{L}_{q}\left(\mathcal{T}_{q}\right)$, contradicting the optimality of $\mathcal{T}_{q}$. Therefore, we must have $d_{1} \leq d_{0}+1$.

A gap in a tree $T$ is a non-empty set of consecutive levels containing only internal nodes of $T$, and such that both the level immediately above the set (assuming the set does not include level 0) and the level immediately below it contain at least one leaf each. The corresponding gap size is defined as the number of levels in the gap. It follows immediately from Lemma 1 that in an optimal tree, if the largest signature above a gap is $s$, then the smallest signature below the gap is $s+1$.

Lemma 2: Let $k=1+\left\lfloor\log q^{-1}\right\rfloor$. Then, for all sufficiently large $s$, the size $g$ of any gap between leaves of signature $s$ and leaves of signature $s+1$ in $\mathcal{T}_{q}$ satisfies $g \leq k-1$.

Proof: We consider the cases $q>\frac{1}{2}, q=\frac{1}{2}$, and $q<\frac{1}{2}$ separately.

Case $q>\frac{1}{2}$. In this case, we have $k=1$, and the claim of the lemma means that there can be no gaps in the tree from a certain level on. Assume that there is a gap between level $d$ with signatures $s$, and level $d^{\prime}$ with signatures $s+1, d^{\prime}-d \geq 2$. By Lemma 1. all signatures $s+1$ are either in level $d^{\prime}$ or in level $d^{\prime}+1$. Without loss of generality, we can assume that there is a subtree of $\mathcal{T}_{q}$ of height at most two, rooted at a node $v$ of depth $d^{\prime}-1 \geq d+1$, and containing at least two leaves of signature $s+1$. Hence, the weight of the subtree satisfies

$$
w(v) \geq 2 q^{s+1}>q^{s},
$$

and switching a leaf $s$ on level $d$ with node $v$ on level $d^{\prime}-1$ decreases the cost of $\mathcal{T}_{q}$, in contradiction with its optimality (when switching nodes, we carry also any subtrees rooted at them). Therefore, there can be no gap between the level containing signatures $s$ and $s+1$, as claimed. Notice that this holds for all values of $s$, regardless of level. 
(A)

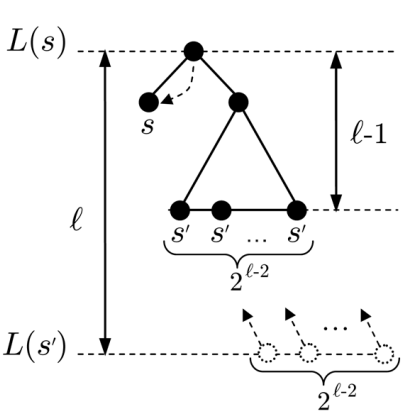

(B)

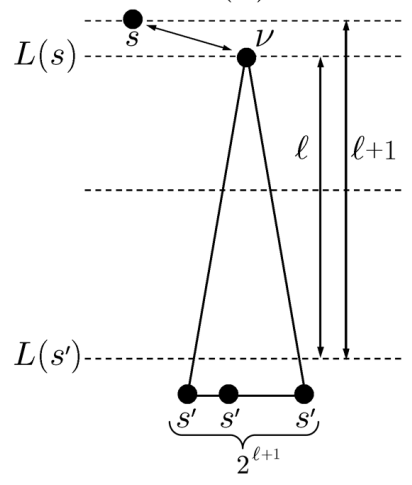

Fig. 1. Tree transformations.

Case $q=\frac{1}{2}$. In this case, the TDGD is dyadic, the optimal profile is uniquely determined, and it and has no gaps (the optimal profile is that of $G_{1} \cdot G_{1}$ ).

Case $q<\frac{1}{2}$. Assume that $s \geq 2^{k}-2$, and that there is a gap of size $g$ between signatures $s$ at level $d$, and signatures $s+1$ at level $d+g+1$. Signatures $s+1$ may also be found at level $d+g+2$. Without loss of generality, and by our assumption on $s$, we can assume that there is a subtree of $\mathcal{T}_{q}$ rooted at a node $v$ at level $d+g+1-k$, and containing at least $2^{k}$ leaves with signature $s+1$, including some at level $d+g+1$. Thus, we have

$$
w(v) \geq 2^{k} q^{s+1}>q^{s}=w(s),
$$

the second inequality following from the definition of $k$. Therefore, we must have $d+g+1-k \leq d$, or equivalently, $g \leq k-1$, for otherwise exchanging $v$ and $s$ would decrease the cost, contradicting the optimality of $\mathcal{T}_{q}$.

Next, we bound the rate of change of signature magnitudes as a function of depth in an optimal tree. Together with the bound on gap sizes in Lemma 2, this will lead to the proof of Theorem 1 It follows from Lemma 1 that for every signature $s \geq 0$ there is a level of $\mathcal{T}_{q}$ containing at least one half of the $s+1$ leaves with signature $s$. We denote the depth of this level by $L(s)$ (with some fixed policy for ties), dependence on $\mathcal{T}_{q}$ being understood from the context.

Lemma 3: Let $s$ be a signature, and $\ell \geq 2$ a positive integer such that $s \geq 2^{\ell+2}-1$, and such that $L\left(s^{\prime}\right)=L(s)+\ell$ for some signature $s^{\prime}>s$. Then, for $\mathcal{T}_{q}$, we have

$$
\frac{\ell-2}{\log q^{-1}} \leq s^{\prime}-s \leq \frac{\ell+1}{\log q^{-1}} .
$$

Proof: Since $s^{\prime}>s \geq 2^{\ell+2}-1>2^{\ell-1}-1$, by the definition of $L\left(s^{\prime}\right)$, there are more than $2^{\ell-2}$ leaves with signature $s^{\prime}$ at level $L\left(s^{\prime}\right)$. We perform the following transformation (depicted in Figure 1(A)) on the tree $\mathcal{T}_{q}$, yielding a modified tree $\mathcal{T}_{q}^{\prime}$ : Choose a leaf with signature $s$ at level $L(s)$, and graft to it a tree with a left subtree consisting of a leaf with signature $s$ ("moved" from the root of the subtree), and a right subtree that is a balanced tree of height $\ell-2$ with $2^{\ell-2}$ leaves of signature $s^{\prime}$. These signatures come from $2^{\ell-2}$ leaves at level $L\left(s^{\prime}\right)$ of $\mathcal{T}_{q}$, which are removed. It is easy to verify that the modified tree $\mathcal{T}_{q}^{\prime}$ defines a valid, albeit incomplete, code for the alphabet of a TDGD. Next, we estimate the change, $\Delta$, in cost due to this transformation. We have

$$
\Delta=\mathcal{L}_{q}\left(\mathcal{T}_{q}^{\prime}\right)-\mathcal{L}_{q}\left(\mathcal{T}_{q}\right)=q^{s}-2^{\ell-2} q^{s^{\prime}} .
$$

The term $q^{s}$ is due to the increase, by one, in the code length for the signature $s$, which causes an increase in cost, while the term $-2^{\ell-2} q^{s^{\prime}}$ is due to the decrease in code length for $2^{\ell-2}$ signatures $s^{\prime}$, which produces a decrease in cost. Since $\mathcal{T}_{q}$ is optimal, we must have $\Delta \geq 0$, namely,

$$
0 \leq q^{s}-2^{\ell-2} q^{s^{\prime}}=q^{s}\left(1-2^{\ell-2} q^{s^{\prime}-s}\right),
$$

and thus, $2^{\ell-2} q^{s^{\prime}-s} \leq 1$, from which the lower bound in (4) follows. (Note: clearly, the condition $s \geq 2^{\ell-1}-1$ would have sufficed to prove the lower bound; the stricter condition of the lemma will be required for the upper bound, and was adopted here for uniformity.)

To prove the upper bound, we apply a different modification to $\mathcal{T}_{q}$. Here, we locate $2^{\ell+1}$ signatures $s^{\prime}$ at level $L\left(s^{\prime}\right)$, and assume, without loss of generality, that these signatures are the leaves of a balanced tree of height $\ell+1$, rooted at a node $\nu$ of depth $L(s)-1$. The availability of the required number of leaves at level $L\left(s^{\prime}\right)$ is guaranteed by the conditions of the lemma. We then exchange $\nu$ with a leaf of signature $s$ at level $L(s)$. The situation, after the transformation, is depicted in Figure 1 (B). The resulting change in cost is computed as follows.

$$
\Delta=\mathcal{L}_{q}\left(\mathcal{T}_{q}^{\prime}\right)-\mathcal{L}_{q}\left(\mathcal{T}_{q}\right)=-q^{s}+2^{\ell+1} q^{s^{\prime}} .
$$

As before, we must have $\Delta \geq 0$, from which the upper bound follows.

We are now ready to prove Theorem 1

Proof of Theorem 7 . We assume, without loss of generality, that $q_{1}>q$, and we write $q_{1}=q(1+\varepsilon), 0<\varepsilon<q^{-1}-1$. In $\mathcal{T}_{q}$, choose a sufficiently large signature $s$ (the meaning of "sufficiently large" will be specified in the sequel), and a node of signature $s$ at level $L(s)$. Let $s^{\prime}>s$ be a signature such that $\ell \triangleq L\left(s^{\prime}\right)-L(s) \geq 2$. We apply the transformation of Figure 1(A) to $\mathcal{T}_{q}$, yielding a modified tree $\mathcal{T}_{q}^{\prime}$. We claim that when weights are taken with respect to $\operatorname{TDGD}\left(q_{1}\right)$, and with an appropriate choice of the parameter $\ell, \mathcal{T}_{q}^{\prime}$ will have strictly lower cost than $\mathcal{T}_{q}$. Therefore, $\mathcal{T}_{q}$ is not optimal for $\operatorname{TDGD}\left(q_{1}\right)$. To prove the claim, we compare the costs of $\mathcal{T}_{q}$ and $\mathcal{T}_{q}^{\prime}$ with respect to $\operatorname{TDGD}\left(q_{1}\right)$. Reasoning as in the proof of the lower bound in Lemma 3, we write

$$
\begin{aligned}
\Delta & =\mathcal{L}_{q_{1}}\left(\mathcal{T}_{q}^{\prime}\right)-\mathcal{L}_{q_{1}}\left(\mathcal{T}_{q}\right)=q_{1}^{s}-2^{\ell-2} q_{1}^{s^{\prime}} \\
& =q_{1}^{s}\left(1-2^{\ell-2} q_{1}^{s^{\prime}-s}\right) \leq q_{1}^{s}\left(1-2^{\ell-2} q_{1}^{\frac{\ell+1}{\log q^{-1}}}\right)
\end{aligned}
$$

where the last inequality follows from the upper bound in Lemma 3 It follows from (5) that we can make $\Delta$ negative if

$$
\ell-2+\frac{\ell+1}{\log q^{-1}} \log q_{1}>0 .
$$

Writing $q_{1}$ in terms of $q$ and $\varepsilon$, and after some algebraic manipulations, the above condition is equivalent to

$$
\ell>3 \frac{\log q^{-1}}{\log (1+\varepsilon)}-1 .
$$


Hence, choosing a large enough value of $\ell$, we get $\Delta<0$, and we conclude that the tree $\mathcal{T}_{q}$ is not optimal for $\operatorname{TDGD}\left(q_{1}\right)$, subject to an appropriate choice of $s$, which we discuss next.

The argument above relies strongly on Lemma 3 . We recall that in order for this lemma to hold, $\ell$ and the signature $s$ must satisfy the condition $s \geq 2^{\ell+2}-1$. Now, it could happen that, after choosing $\ell$ according to (6) and then $s$ according to the condition of Lemma 3 , the level $L(s)+\ell$ does not contain $2^{\ell-2}$ signatures $s^{\prime}$ as required (e.g., when the level is part of a gap). This would force us to increase $\ell$, which could then make $s$ violate the condition of the lemma. We would then need to increase $s$, and re-check $\ell$, in a potentially vicious circle. The bound on gap sizes of Lemma 2 allows us to avoid this trap. The bound in the lemma depends only on $q$ and thus, for a given TDGD, it is a constant, say $g_{q}$. Thus, first, we choose a value $\ell_{0}$ satisfying the constraint on $\ell$ in 6 . Then, we choose $s \geq 2^{\ell_{0}+g_{q}+4}$. Now, we try $\ell=\ell_{0}, \ell_{0}+1, \ell_{0}+2, \ldots$, in succession, and check whether level $L(s)+\ell$ contains enough of the required signatures. By Lemmas 1 and 2, an appropriate level $L\left(s^{\prime}\right)$ will be found for some $\ell \leq \ell_{0}+g_{q}+2$. For such a value of $\ell$, we have $2^{\ell+2}-1 \leq 2^{\ell_{0}+g_{q}+4}-1<s$, satisfying the condition of Lemma 3 This condition, in turn, guarantees also that there are at least $2^{\ell-2}$ signatures $s^{\prime}$ at $L\left(s^{\prime}\right)$, as required.

\section{OPTIMAL CODES FOR TDGDS WITH $q=2^{-1 / k}$}

It follows from the results of Section III that it is infeasible to provide a compact description of optimal codes for TDGDs covering all values of the parameter $q$, as can be done with one-dimensional geometric distributions [1], [2] or their twosided variants [3]. Instead, we describe optimal prefix codes for a discrete sequence of values of $q$, which provide good coverage of the parameter range. In this section, we study optimal codes for TDGDs with parameters $q=2^{-1 / k}$ for integers $k \geq 1$, i.e., $q \geq \frac{1}{2}$, while in Section $\mathrm{V}$ we consider parameters of the form $q=2^{-k}, k>1$, covering the range $q<\frac{1}{2}$ (the two parameter sequences coincide at $k=1, q=\frac{1}{2}$, which we choose to assign to the case covered in this section).

\section{A. Initial characterization of optimal codes for $q=2^{-1 / k}$}

The following theorem characterizes optimal codes for TDGDs of parameter $q=2^{-1 / k}, k \geq 1$, in terms of unary codes and Huffman codes for certain finite distributions. In Subsection IV-C we further refine the characterization by providing explicit descriptions of these Huffman codes.

Theorem 2: An optimal prefix code $C_{k}$ for $\operatorname{TDGD}(q)$, with $q=2^{-1 / k}, k \geq 1$, is given by

$$
C_{k}(i, j)=T_{k}(i \bmod k, j \bmod k) \cdot G_{1}\left(\left\lfloor\frac{i}{k}\right\rfloor\right) \cdot G_{1}\left(\left\lfloor\frac{j}{k}\right\rfloor\right),
$$

where $G_{1}$ is the unary code, and $T_{k}$, referred to as the top code, is an optimal code for the finite source defined by the following symbol set and respective weights:

$$
\hat{\mathcal{A}}_{k}=\{(i, j) \mid 0 \leq i, j<k\}, \quad w(i, j)=q^{i+j} .
$$

\section{Remarks.}

1) Theorem 2 can readily be generalized to blocks of $d>2$ symbols. For simplicity, we present the proof for $d=2$.
(A)

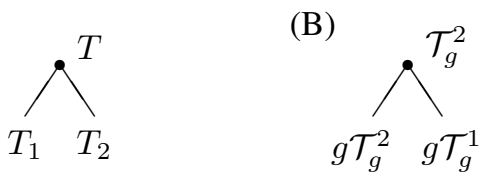

Fig. 2. Graphical representations for trees with associated weights.

2) Notice that $C_{k}(i, j)$ concatenates the "unary" parts of the codewords for $i$ and $j$ in a Golomb code of order $k$ (as if encoding $i$ and $j$ separately), but encodes the "binary" part jointly by means of $T_{k}$, which, in general, does not yield the concatenation of the respective "binary" parts $Q_{k}(i)$ and $Q_{k}(j)$. However, when $k=1$ and $k=2, C_{k}$ is equivalent to the full concatenation $G_{k} \cdot G_{k}$. When $k=1$, the code $T_{k}$ is void, and $C_{1}=G_{1} \cdot G_{1}$. The parameter in this case is $q=\frac{1}{2}$, the geometric distribution is dyadic, and the code redundancy is zero. When $k=2$, we have $q=1 / \sqrt{2}$ and the finite source $\hat{\mathcal{A}}_{k}$ has four symbols with respective weights $\{1, \sqrt{2} / 2, \sqrt{2} / 2,1 / 2\}$. This source is quasi-uniform, and, therefore, it admits $Q_{4}$ as an optimal tree. This is a balanced tree of depth two, which can also be written as $Q_{4}=Q_{2} \cdot Q_{2}$. Thus, we have $C_{2}=G_{2} \cdot G_{2}$. Later on in the section, in Corollary 1, we will show that this situation will not repeat for larger values of $k$ : the "symbol by symbol" code $G_{k} \cdot G_{k}$ is strictly suboptimal for $\operatorname{TDGD}\left(2^{-1 / k}\right)$ when $k>2$.

In deriving the proof of Theorem 2 and in subsequent sections, we shall make use of the following notations to describe and operate on some infinite trees with weights associated to their leaves. We denote by $v$ the trivial tree consisting of a single node (leaf) of weight $v$. Given a tree $T$ and a scalar $g, g T$ denotes the tree $T$ with all its weights multiplied by $g$. Given trees $T_{1}$ and $T_{2}$, the graphic notation in Figure 2(A) represents a tree $T$ consisting of a root node with $T_{1}$ as its left subtree and $T_{2}$ as its right subtree, each contributing its respective leaf weights. The multiset of weights associated with $T$ is the union of the multisets associated with $T_{1}$ and $T_{2}$. We will also use the notation $\left[\begin{array}{ll}T_{1} & T_{2}\end{array}\right]$ to represent the forest consisting of the separate trees $T_{1}$ and $T_{2}$, which has the same associated multiset of weights as the tree $T$ of Figure 2(A), but a different underlying graph. We denote by $\mathcal{T}_{g}^{1}$ the tree of a unary code whose leaf at each depth $i \geq 1$ has weight $g^{i}$, and by $\mathcal{T}_{g}^{2}$ the structure in Figure 2(B). It is readily verified that $\mathcal{T}_{g}^{2}$ corresponds to the concatenation of two unary codes, with each of the $i-1$ leaves at depth $i \geq 2$ of $\mathcal{T}_{g}^{2}$ carrying weight $g^{i}$. In particular, as shown in Figure 3, the tree $q^{-2} \mathcal{T}_{q}^{2}$ corresponds to the optimal tree for the dyadic TDGD with $q=\frac{1}{2}$, where each leaf is weighted according to the signature of the symbol it encodes.

The following lemma follows directly from the above definitions, applying elementary symbolic manipulations on geometric sums.

Lemma 4: For any real number $g$, $0<g<1$, we have $w\left(\mathcal{T}_{g}^{2}\right)=w\left(\mathcal{T}_{g}^{1}\right)^{2}=\left(\frac{g}{1-g}\right)^{2}$. In particular, if $q=2^{-1 / k}$, 


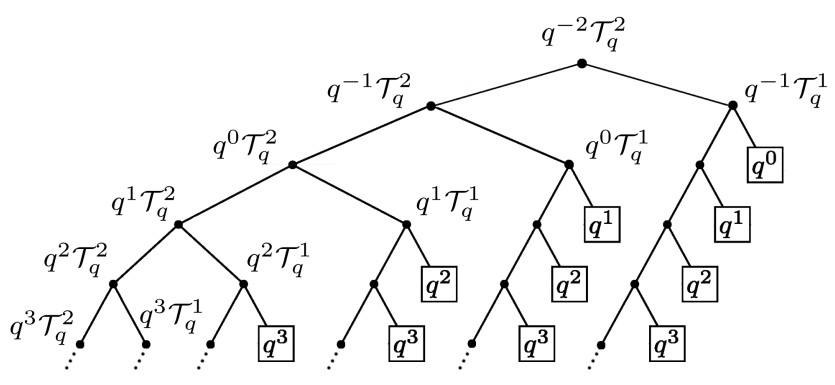

Fig. 3. The tree $q^{-2} \mathcal{T}_{q}^{2}$.

we have $w\left(\mathcal{T}_{q^{k}}^{2}\right)=w\left(\mathcal{T}_{q^{k}}^{1}\right)=1$.

We rely on this observation in the proof of Theorem 2 below. In the proof, when defining virtual symbols, we further overload notation and regard trees with associated weights, such as $q^{r} \mathcal{T}_{q^{k}}^{d}$, also as multisets of signatures, with a signature $s$ for each leaf of the tree with weight $q^{s}$.

Proof of Theorem 2 2 We use the Gallager-Van Voorhis construction [2]. For $s \geq 0$, define the reduced source

$$
\mathcal{W}_{s}=\mathcal{H}_{s} \cup \mathcal{F}_{s}
$$

where

$$
\mathcal{H}_{s}=\{i \in \hat{\mathcal{A}} \mid i<s\}
$$

(signatures in $\mathcal{H}_{s}$ occur with the same multiplicity as in $\hat{\mathcal{A}}$ ), and

$$
\mathcal{F}_{s}=\bigcup_{i=0}^{k-1}\{\underbrace{q^{s+i} \mathcal{T}_{q^{k}}^{2}}_{k \text { times }}, \underbrace{q^{s+i} \mathcal{T}_{q^{k}}^{1}}_{\begin{array}{c}
s+k+i+1 \\
\text { times }
\end{array}}, \underbrace{s+i}_{\begin{array}{c}
s+i+1 \\
\text { times }
\end{array}}\} .
$$

The multisets (of signatures) $q^{s+i} \mathcal{T}_{q^{k}}^{1}$ and $q^{s+i} \mathcal{T}_{q^{k}}^{2}$ play the role of virtual symbols in the reduced sources, as discussed in Subsection II-C (we omit the qualifier 'virtual' in the sequel). It is readily verified that all the weights of symbols in $\mathcal{F}_{s}$ are smaller than the weights of signatures in $\mathcal{H}_{s}$. Since $q=2^{-1 / k}$, by Lemma 4 we have $w\left(q^{s+i} \mathcal{T}_{q^{k}}^{2}\right)=w\left(q^{s+i} \mathcal{T}_{q^{k}}^{1}\right)=w(s+i)$. Thus, we can apply steps of the Huffman procedure to $\mathcal{F}_{s}$ in such way that the $s+i+1$ signatures $s+i$ are merged with $s+i+1$ symbols $q^{s+i} \mathcal{T}_{q^{k}}^{1}$, resulting in $s+i+1$ trees $q^{s+i-k} \mathcal{T}_{q^{k}}^{1}$. The remaining $k$ symbols $q^{s+i} \mathcal{T}_{q^{k}}^{1}$ can be merged with the $k$ symbols $q^{s+i} \mathcal{T}_{q^{k}}^{2}$, resulting in $k$ trees $q^{s+i-k} \mathcal{T}_{q^{k}}^{2}$ when $i$ ranges from $k-1$ down to 0 . After this sequence of Huffman mergers, $\mathcal{W}_{s}$ is transformed into $\mathcal{W}_{s-k}$, as long as $s \geq k$. Starting from $s=t k$ for some $t>0$, the procedure eventually leads to $\mathcal{W}_{0}$. Formally, our reduced source $\mathcal{W}_{t k}, t \geq 0$, corresponds to $\mathcal{S}_{t}$ in our description of the Gallager-Van Voorhis construction in Section II-C. Thus, the iteration leads to $\mathcal{S}_{0}$, as called for in the construction. It is readily verified that this source admits an additional sequence of Huffman mergers, as described above, leading (with a slight abuse of notation) to

$$
\mathcal{S}_{-1}=\bigcup_{i=0}^{k-1}\{\underbrace{q^{i-k} \mathcal{T}_{q^{k}}^{2}}_{\substack{k \\ \text { times }}}, \underbrace{q^{i-k} \mathcal{T}_{q^{k}}^{1}}_{\substack{i+1 \\ \text { times }}}\} .
$$

Continuing with the Huffman procedure, each symbol $q^{i-k} \mathcal{T}_{q^{k}}^{1}$ in $\mathcal{S}_{-1}$ can be merged with a symbol $q^{i-k} \mathcal{T}_{q^{k}}^{2}$, further leading, by the definition of $\mathcal{T}_{g}^{2}$ (see Figure 2 (B)), to a reduced source

$$
\begin{aligned}
& \mathcal{S}^{*}=\{\underbrace{q^{-2 k} \mathcal{T}_{q^{k}}^{2}}_{\text {time }}, \underbrace{q^{-2 k+1} \mathcal{T}_{q^{k}}^{2}}_{\begin{array}{c}
2 \\
\text { times }
\end{array}}, \underbrace{q^{-2 k+2} \mathcal{T}_{q^{k}}^{2}}_{\begin{array}{c}
3 \\
\text { times }
\end{array}}, \ldots \\
& \ldots, \underbrace{q^{-k-1} \mathcal{T}_{q^{k}}^{2}}_{\substack{k \\
\text { times }}}, \underbrace{q^{-k} \mathcal{T}_{q^{k}}^{2}}_{\substack{k-1 \\
\text { times }}}, \ldots, \underbrace{q^{-3} \mathcal{T}_{q^{k}}^{2}}_{\substack{2 \\
\text { times }}}, \underbrace{q^{-2} \mathcal{T}_{q^{k}}^{2}}_{\begin{array}{c}
1 \\
\text { time }
\end{array}}\} .
\end{aligned}
$$

We now take a common "factor" $q^{-2 k} \mathcal{T}_{q^{k}}^{2}$ from each symbol of $\mathcal{S}^{*}$. By the discussion of Figures 2 and 3 , this factor corresponds to a copy of $G_{1} \cdot G_{1}$, with weights that get multiplied by $q^{k}$ every time the depth increases by 1 . After the common factor is taken out, the source $\mathcal{S}^{*}$ becomes the source $\hat{\mathcal{A}}_{k}$ of (7), to which the Huffman procedure needs to be applied to complete the code construction. Thus, the code described in the theorem is optimal.

To make the result of Theorem 2 completely explicit, it remains to characterize an optimal prefix code for the finite source $\hat{\mathcal{A}}_{k}$ of 7 . The following lemma presents some basic properties of $\mathcal{A}_{k}$ and its optimal trees. Recall the definitions of $\alpha$-uniformity and fringe thickness from Section III.

Lemma 5: The source $\hat{\mathcal{A}}_{k}$ is 4-uniform, and it has an optimal tree $T$ of fringe thickness $f_{T} \leq 2$.

Proof: It follows from (7) and the relation $q^{k}=\frac{1}{2}$ that the maximal ratio between weights of symbols in $\hat{\mathcal{A}}_{k}$ is $q^{-2 k+2}=$ $4 q^{2}<4$. Hence, $\hat{\mathcal{A}}_{k}$ is 4 -uniform. The claim on the optimal tree holds trivially for $k \leq 2$, in which case the optimal tree for $\hat{\mathcal{A}}_{k}$ is uniform. To prove the claim for $k>2$, consider the multiset $\hat{\mathcal{A}}_{k}^{*} \subseteq \hat{\mathcal{A}}_{k}$ consisting of the lightest $2\left\lceil\frac{k(k-1)}{4}\right\rceil$ signatures in $\hat{\mathcal{A}}_{k}$, i.e.,

$$
\begin{aligned}
\hat{\mathcal{A}}_{k}^{*}=\mathcal{K} \cup\{\underbrace{k, k, \ldots, k}_{k-1 \text { times }}, \underbrace{k+1, \ldots, k+1}_{k-2 \text { times }}, \ldots \\
\ldots, \underbrace{2 k-3,2 k-3}_{2 \text { times }}, \underbrace{2 k-2}_{1 \text { time }}\},
\end{aligned}
$$

where $\mathcal{K}=\{k-1\}$ if $k \bmod 4 \in\{2,3\}$, or $\mathcal{K}$ is empty otherwise. The sum of the two smallest weights of signatures in $\hat{\mathcal{A}}_{k}^{*}$ satisfies

$$
\begin{aligned}
w(2 k-2)+w(2 k-3) & =q^{2 k-2}+q^{2 k-3}=q^{2 k-2}\left(1+q^{-1}\right) \\
& =\frac{1}{2}\left(1+q^{-1}\right) q^{k-2}>w(k-2) .
\end{aligned}
$$

The sum of the two largest weights in $\hat{\mathcal{A}}_{k}^{*}$, on the other hand, is either $q^{0}$ if $k \bmod 4 \in\{0,1\}$, or $\frac{1}{2}\left(1+q^{-1}\right)$ otherwise. Therefore, if the Huffman procedure is applied to $\hat{\mathcal{A}}_{k}$, every pair of consecutive elements of $\hat{\mathcal{A}}_{k}^{*}$ will be merged, without involving a previously merged pair. The ratio of the largest to the smallest weight remaining after these mergers is at most $\frac{1}{2}\left(1+q^{-1}\right) / q^{k-1}=q+1<2$. Hence, the resulting source is quasi-uniform and has a quasi-uniform optimal tree. Therefore, completing the Huffman procedure for $\hat{\mathcal{A}}_{k}$ results in an optimal tree of fringe thickness at most two.

To complete the explicit description of an optimal tree for $\hat{\mathcal{A}}_{k}$, we will rely on a characterization of trees $T$ with $f_{T} \leq 2$ 
that are optimal for 4 -uniform sources ${ }^{5}$ This characterization is presented next.

\section{B. Optimal trees with $f_{T} \leq 2$ for 4-uniform sources}

To proceed as directly as possible to the construction of an optimal tree for $\hat{\mathcal{A}}_{k}$, we defer all the proofs of results in this subsection to Appendix A. We start by characterizing all the possible profiles for a tree $T$ with $N$ leaves, and $f_{T} \leq 2$. Let $T$ be such a tree, let $m=\lceil\log N\rceil$, and denote by $n_{\ell}$ the number of leaves at depth $\ell$ in $T$.

Lemma 6: The profile of $T$ satisfies $n_{\ell}=0$ for $\ell<m-2$ and $\ell>m+1$, and either $n_{m-2}=0$ or $n_{m+1}=0$ (or both, when $f_{T} \leq 1$ ).

It follows from Lemma 6 that $T$ is fully characterized by the quadruple $\left(n_{m-2}, n_{m-1}, n_{m}, n_{m+1}\right)$, with either $n_{m-2}=0$ or $n_{m+1}=0$. We say $T$ is long if $n_{m-2}=0$, and that $T$ is short if $n_{m+1}=0$. Defining $M=m-\sigma$, where $\sigma=1$ if $T$ is short, or 0 if it is long, a tree with $f_{T} \leq 2$ can be characterized more compactly by a triple of nonnegative integers $\mathbf{N}_{T}=$ $\left(n_{M-1}, n_{M}, n_{M+1}\right)$. We will also refer to this triple as the (compact) profile of $T$, with the associated parameters $N, m$, and $\sigma$ understood from the context. Notice that when $n_{m-2}=$ $n_{m+1}=0, T$ is the quasi-uniform tree $Q_{N}$, and (abusing the metaphor), it is considered both long and short (i.e., it has representations with both $\sigma=0$ and $\sigma=1$ ).

Lemma 7: Let $T$ be a tree with $f_{T} \leq 2$. For $\sigma \in\{0,1\}$ and $M=m-\sigma$, define

$$
\underline{c}_{\sigma}=\left(N-2^{M}\right) \sigma \quad \text { and } \quad \bar{c}_{\sigma}=\left\lfloor\frac{2 N-2^{M}}{3}\right\rfloor .
$$

Then, $T$ is equivalent to one of the trees $T_{\sigma, c}$ defined by the profiles

$$
\begin{aligned}
\mathbf{N}_{T_{\sigma, c}}= & \left(n_{M-1}, n_{M}, n_{M+1}\right) \\
= & \left(2^{M}-N+c, 2 N-2^{M}-3 c, 2 c\right), \\
& \sigma \in\{0,1\}, \underline{c}_{\sigma} \leq c \leq \bar{c}_{\sigma} .
\end{aligned}
$$

\section{Remarks.}

1) Equation (8) characterizes all trees with $N$ leaves and $f_{T} \leq 2$ in terms of the parameters $\sigma$ and $c$. The parameter $c$ has different ranges depending on $\sigma$ : we have $N-2^{m-1} \leq c \leq\left\lfloor\frac{2 N-2^{m-1}}{3}\right\rfloor$ when $\sigma=1$, and $0 \leq c \leq\left\lfloor\frac{2 N-2^{m}}{3}\right\rfloor$ when $\sigma=0$. The use of the parametrized quantities $M, \underline{c}_{\sigma}$, and $\bar{c}_{\sigma}$ will allow us to treat the two ranges in a unified way in most cases. Also, notice that $T_{1, c_{1}}$ and $T_{0, c_{0}}$ represent the same tree, corresponding, respectively, to interpretations of the quasi-uniform tree $Q_{N}$ as short or long.

2) The parameter $c$ represents the number of internal (nonleaf) nodes at level $M$ of $T$. An increase of $c$ by one corresponds to moving a pair of sibling leaves previously rooted at level $M-1$ to a new parent at level $M$ (thereby increasing the number of internal nodes at that level by

\footnotetext{
${ }^{5}$ Notice that not every 4-uniform source admits an optimal tree with $f_{T} \leq 2$ (although the ones of interest in this section do). For example, an optimal tree for the 4-uniform source with probabilities $\frac{1}{10}(4,3,1,1,1)$ must have $f_{T}>2$.
}

one). The number of leaves at level $M$ decreases by three, and the numbers of leaves at levels $M-1$ and $M+1$ increase by one and two, respectively.

Consider now a distribution on $N$ symbols, with associated vector of probabilities (or weights) $\mathbf{p}=\left(p_{1}, p_{2}, \ldots, p_{N}\right)$, $p_{1} \geq p_{2} \geq \cdots \geq p_{N}$. Let $L_{\sigma, c}$ denote the average code length of $T_{\sigma, c}$ under $\mathbf{p}$ (with shorter codewords naturally assigned to larger weights), and let

$$
D_{\sigma, c}=L_{\sigma, c}-L_{\sigma, c-1}, \quad \sigma \in\{0,1\}, \quad \underline{c}_{\sigma}<c \leq \bar{c}_{\sigma} .
$$

It follows from these definitions, and the structure of the profile (8) (see also Remark 2 above), that for $\sigma \in\{0,1\}$ and $\underline{c}_{\sigma}<c \leq \bar{c}_{\sigma}$, we have

$$
D_{\sigma, c}=p_{N-2 c+1}+p_{N-2 c+2}-p_{2^{M}-N+c} .
$$

A useful interpretation of 10 follows directly from the profile (8): for $T_{\sigma, c}, D_{\sigma, c}$ is the difference between the sum of the two heaviest weights on level $M+1$ and the lightest weight on level $M-1$.

Let $\operatorname{sg}(x)$ be defined as $-1,0$, or 1 , respectively, for negative, zero, or positive values of $x$, and consider the following sequence (recalling that $\underline{c}_{0}=0$ ):

$$
\begin{aligned}
\mathbf{s}=- & \operatorname{sg}\left(D_{1, \bar{c}_{1}}\right),-\operatorname{sg}\left(D_{1, \bar{c}_{1}-1}\right), \ldots,-\operatorname{sg}\left(D_{1, \underline{c}_{1}+1}\right), \\
& \operatorname{sg}\left(D_{0,1}\right), \operatorname{sg}\left(D_{0,2}\right), \ldots, \operatorname{sg}\left(D_{0, \bar{c}_{0}}\right) .
\end{aligned}
$$

Lemma 8: The sequence $\mathbf{s}$ is non-decreasing.

The definition of the sequence $\mathbf{s}$ induces a total ordering of the pairs $(\sigma, c)$ (and, hence, also of the trees $\left.T_{\sigma, c}\right)$, with pairs with $\sigma=1$ ordered by decreasing value of $c$, followed by pairs with $\sigma=0$ in increasing order of $c$. The two subsequences "meet" at $\underline{c}_{\sigma}$, which defines the same tree regardless of the value of $\sigma$ (in the pairs ordering, we take $\left(1, \underline{c}_{1}\right)$ as identical to $\left.\left(0, \underline{c}_{0}\right)=(0,0)\right)$. We denote this total order by $\preceq$. Recalling that the quantities $D_{\sigma, c}$ are differences in average code length between consecutive codes in this ordering, Lemma 8 tells us that, as we scan the codes in order, we will generally see the average code length decrease monotonically, reach a minimum, and then (possibly after staying at the minimum for some number of trees) increase monotonically. In the following theorem, we formalize this observation, and identify the trees $T_{\sigma, c}$ that are optimal for $\mathbf{p}$.

Theorem 3: Let $\mathbf{p}$ be a 4-uniform distribution such that $\mathbf{p}$ has an optimal tree $T$ with $f_{T} \leq 2$. Define pairs $\left(\sigma_{*}, c_{*}\right)$ and $\left(\sigma^{*}, c^{*}\right)$ as follows:

$$
\begin{aligned}
& \left(\sigma_{*}, c_{*}\right)=\left(1, \bar{c}_{1}\right) \text { if } D_{1, \bar{c}_{1} \geq 0,}, \\
& \left(\sigma^{*}, c^{*}\right)=\left(0, \bar{c}_{0}\right) \text { if } D_{0, \bar{c}_{0}} \leq 0 ;
\end{aligned}
$$

otherwise, if $D_{1, \bar{c}_{1}}<0$, let $\left(\sigma_{-}, c_{-}\right)$be such that $(-1)^{\left(\sigma_{-}\right)} \operatorname{sg}\left(D_{\sigma_{-}, c_{-}}\right)$is the last negative entry in $\mathbf{s}$, and define

$$
\left(\sigma_{*}, c_{*}\right)=\left(\sigma_{-}, c_{-}-\sigma_{-}\right) ;
$$

if $D_{0, \bar{c}_{0}}>0$, let $\left(\sigma_{+}, c_{+}\right)$be such that $(-1)^{\left(\sigma_{+}\right)} \operatorname{sg}\left(D_{\sigma_{+}, c_{+}}\right)$is the first positive entry in $\mathbf{s}$, and define

$$
\left(\sigma^{*}, c^{*}\right)=\left(\sigma_{+}, c_{+}-1+\sigma_{+}\right) .
$$

Then, all trees $T_{\sigma, c}$ with $\left(\sigma_{*}, c_{*}\right) \preceq(\sigma, c) \preceq\left(\sigma^{*}, c^{*}\right)$ are optimal for $\mathbf{p}$. 
TABLE I

Finding OPtimAL TREES $T_{\sigma, c}$ FOR $N=19, \mathbf{p}=\frac{1}{49}(4,4,3,3,3,3,3,3,3,3,3,2,2,2,2,2,2,1,1)$ (OPTIMAL TREE PARAMETERS EMPHASIZED IN BOLDFACE).

\begin{tabular}{|r|ccccccc|}
\hline$(\sigma, c)$ & $(1,7)$ & $(1,6)$ & $(1,5)$ & $(\mathbf{1}, \mathbf{4})$ & $\begin{array}{c}(\mathbf{1}, \mathbf{3})= \\
\mathbf{0}, \mathbf{0})\end{array}$ & $(\mathbf{0}, \mathbf{1})$ & $(0,2)$ \\
\hline$\left(n_{M-1}, n_{M}, n_{M+1}\right)$ & $(4,1,14)$ & $(3,4,12)$ & $(2,7,10)$ & $(\mathbf{1}, \mathbf{1 0}, \mathbf{8})$ & $(\mathbf{1 3}, \mathbf{6}, \mathbf{0})$ & $(\mathbf{1 4}, \mathbf{3}, \mathbf{2})$ & $(15,0,4)$ \\
\hline $49 \cdot L_{\sigma, c}$ & 214 & 211 & 208 & $\mathbf{2 0 6}$ & $\mathbf{2 0 6}$ & $\mathbf{2 0 6}$ & 208 \\
\hline $49 \cdot D_{\sigma, c}$ & 3 & 3 & 2 & 0 & & 0 & 2 \\
\hline $\mathbf{S}$ & -1 & -1 & $\left(\sigma_{-}^{-1}, c_{-}\right)$ & $\left(\sigma_{*}, c_{*}\right)$ & & $\left(\sigma^{*}, c^{*}\right)$ & $\left(\sigma_{+}, c_{+}\right)$ \\
\hline
\end{tabular}

Notice that, by Lemma 8 , the range $\left(\sigma_{*}, c_{*}\right) \preceq(\sigma, c) \preceq$ $\left(\sigma^{*}, c^{*}\right)$ is well defined and never empty, consistently with the assumptions of the theorem and with Lemma 7 . The example in Table $\mathrm{I}$ lists all the trees $T_{\sigma, c}$ with $f_{T} \leq 2$ for $N=19$, as characterized in Lemma 7, and shows how Theorem 3 is used to find optimal trees for a given 4-uniform distribution on 19 symbols.

\section{The top code}

By Lemma 5 , Theorem 3 applies to the source $\hat{\mathcal{A}}_{k}$ defined in (7). We will apply the theorem to identify parameters $\left(\sigma_{k}, c_{k}\right)$ that yield an optimal tree $T_{\sigma_{k}, c_{k}}$ for $\hat{\mathcal{A}}_{k}$.

For the remainder of the section, we take $N=k^{2}$, and let $\mathbf{p}=\left(p_{1}, p_{2}, \ldots, p_{k^{2}}\right)$ denote the vector of (unnormalized) symbol weights in $\hat{\mathcal{A}}_{k}$, in non-increasing order. Thus, we have $\mathbf{p}=\left(q^{0}, q^{1}, q^{1}, \ldots, q^{j}, q^{j}, \ldots, q^{j}, \ldots, q^{2 k-3}, q^{2 k-3}, q^{2 k-2}\right)$. Here, $q^{j}$ is repeated $j+1$ times for $0 \leq j \leq k-1$, and $2 k-1-j$ times for $k \leq j \leq 2 k-2$. The following lemma, which follows immediately from this structure, establishes the relation between indices and weights in $\mathbf{p}$.

Lemma 9: For $0 \leq i<k(k+1) / 2$, we have $p_{i+1}=q^{j}$, where $j$ is the unique integer in the range $0 \leq j \leq k-1$ satisfying

$$
i=\frac{j(j+1)}{2}+r \quad \text { for some } r, \quad 0 \leq r \leq j .
$$

For $0 \leq i^{\prime}<k(k+1) / 2$, we have $p_{k^{2}-i^{\prime}}=q^{2 k-2-j^{\prime}}=$ $\frac{1}{2} q^{k-2-j^{\prime}}$, where $j^{\prime}$ is the unique integer in the range $0 \leq$ $j^{\prime} \leq k-1$ satisfying

$$
i^{\prime}=\frac{j^{\prime}\left(j^{\prime}+1\right)}{2}+r^{\prime} \quad \text { for some } r^{\prime}, \quad 0 \leq r^{\prime} \leq j^{\prime} .
$$

We define some auxiliary quantities that will be useful in the sequel. Let $m=\left\lceil\log k^{2}\right\rceil, Q=k^{2}-\lceil k(k-1) / 4\rceil$, and $M^{\prime}=$ $\left\lceil\log _{2} Q\right\rceil$, with dependence on $k$ understood from the context. We assume that $k>2$, since the optimal codes for $k=1$ and $k=2$ have already been described in Subsection IV-A It is readily verified that we must have either $M^{\prime}=m$ or $M^{\prime}=m-1$. The next lemma shows that the relation between $M^{\prime}$ and $m$ determines the parameter $\sigma$ of the optimal trees $T_{\sigma, c}$ for $\hat{\mathcal{A}}_{k}$.

Lemma 10: If $M^{\prime}=m$, then trees $T_{\sigma, c}$ that are optimal for $\hat{\mathcal{A}}_{k}$ are long $(\sigma=0)$; otherwise, they are short $(\sigma=1)$.

Proof: Assume $M^{\prime}=m$. Then, we can write

$$
\begin{aligned}
2^{m} & =2^{M^{\prime}}<2^{1+\log Q}=2 Q \\
& =2 k^{2}-2\lceil k(k-1) / 4\rceil \leq 2 k^{2}-k(k-1) / 2,
\end{aligned}
$$

so $2^{m}-k^{2}<k^{2}-k(k-1) / 2$. If $\underline{c}_{1}+1>\bar{c}_{1}$, then all trees $T_{\sigma, c}$ in 8 are long. Otherwise, $D_{1, \underline{c}_{1}+1}$ is well defined, and we have

$$
\begin{aligned}
& -D_{1, \underline{c}_{1}+1}=-D_{1, k^{2}-2^{m-1}+1} \\
& \quad=p_{1}-\left(p_{2^{m}-k^{2}-1}+p_{2^{m}-2^{k}}\right) \\
& \quad \leq p_{1}-2 p_{k^{2}-k(k-1) / 2}=p_{1}-2 q^{k-1}=1-q^{-1}<0,
\end{aligned}
$$

where the first and second equalities follow from the definition of $\underline{c}_{1}$ and from $(10)$, the first inequality from the ordering of the weights and from (14), the third equality from Lemma 9 . and the last equality from the relation $q^{k}=\frac{1}{2}$. By Lemma 8 we conclude that optimal trees for $\hat{\mathcal{A}}_{k}$ are long in this case. Similarly, when $M^{\prime}=m-1$, we have

$$
2^{m} \geq 2 Q \geq 2 k^{2}-k(k-1) / 2-2,
$$

so $2^{m}-k^{2}+1 \geq k^{2}-k(k-1) / 2-1$, and $p_{2^{m}-k^{2}+1} \leq$ $p_{k^{2}-k(k-1) / 2-1}=q^{k}=\frac{1}{2}$. If $\bar{c}_{0}=\underline{c}_{0}=0$, then all trees $T_{\sigma, c}$ in (8) are short. Otherwise, similarly to (15), we have

$D_{0,1}=p_{k^{2}-1}+p_{k^{2}}-p_{2^{m}-k^{2}+1}>2 q^{2 k-2}-\frac{1}{2}=\frac{q^{-2}}{2}-\frac{1}{2}>0$, which implies that optimal trees are short in this case.

It follows from Lemma 10 that we can take $m-M^{\prime}$ as the parameter $\sigma$ for all trees $T_{\sigma, c}$ that are optimal for $\mathbf{p}$. Notice that $M^{\prime}$ is analogous to the parameter $M$ defined in Lemma 7, but slightly stricter, in that, in cases where a quasi-uniform tree is optimal, $m-M^{\prime}$ will assume a definite value in $\{0,1\}$ (which will vary with $k$ ), while, in principle, a representation with either value of $\sigma$ is available. This very slight loss of generality is of no consequence to our derivations, and, in the sequel, we will identify $M$ with $M^{\prime}$, i.e., we will take $M=\lceil\log Q\rceil$. It also follows from Lemma 10 that when applying Theorem 3 to find optimal trees for $\mathbf{p}$, we only need to focus on one of the two segments (corresponding to $\sigma=0$ or $\sigma=1$ ) that comprise the sequence $\mathrm{s}$ in (11), the choice being determined by the value of $k$. This will simplify the application of the theorem.

Lemmas 9 and 10, together with Theorem 3, suggest a clear way, at least in principle, for finding an optimal tree $T_{\sigma, c}$ for $\hat{\mathcal{A}}_{k}$. The parameter $\sigma$ is determined immediately as $\sigma=m-M$ (recalling that $m$ and $M$ are determined by $k$ ). Now, recalling the expression for $D_{\sigma, c}$ in $(10)$, we observe that as $c$ increases, the weights $p_{k^{2}-2 c+1}$ and $p_{k^{2}-2 c+2}$ also increase, while $p_{2^{M}-k^{2}+c}$, which gets subtracted, decreases. Thus, since, by Theorem 3 , an optimal value of $c$ occurs when $D_{\sigma, c}$ changes sign, we need to search for the value of $c$ for 
TABLE II

OPTIMAL CODE PARAMETERS AND PROFILES FOR $\hat{\mathcal{A}}_{k}, 3 \leq k \leq 10$

\begin{tabular}{|rrrrrrc|}
\hline$k$ & $M$ & $j$ & $r$ & $\sigma_{k}$ & $c_{k}$ & $\left(n_{M-1}, n_{M}, n_{M+1}\right)$ \\
\hline 2 & 2 & 0 & 0 & 0 & 0 & $(0,4,0)$ \\
3 & 3 & 0 & 0 & 1 & 1 & $(0,7,2)$ \\
4 & 4 & 1 & 0 & 0 & 1 & $(1,13,2)$ \\
5 & 5 & 3 & 1 & 0 & 0 & $(7,18,0)$ \\
6 & 5 & 1 & 0 & 1 & 5 & $(1,25,10)$ \\
7 & 6 & 5 & 0 & 0 & 0 & $(15,34,0)$ \\
8 & 6 & 2 & 2 & 0 & 5 & $(5,49,10)$ \\
9 & 6 & 0 & 0 & 1 & 17 & $(0,47,34)$ \\
10 & 7 & 7 & 1 & 0 & 1 & $(29,69,2)$ \\
\hline
\end{tabular}

which the increasing sum of the first two terms "crosses" the value of the decreasing third term. This can be done, at least roughly, by using explicit weight values from Lemma 9 with $i^{\prime} \in\{2 c-1,2 c-2\}$ and $i=2^{m}-k^{2}+c$, and solving a quadratic equation, say, for the parameter $j$ (the parameter $j^{\prime}$ will be tied to $j$ by the constraint $D_{\sigma, c} \approx 0$ ). A finer adjustment of the solution is achieved with the parameters $r$ and $r^{\prime}$, observing that a change of sign of $D_{\sigma, c}$ can only occur near locations where the weights in $\mathbf{p}$ change (i.e., "jumps" in either $j$ or $j^{\prime}$ ), which occur at intervals of length up to $k$. At the "jump" locations, either $r$ or $r^{\prime}$ must be close to zero. While there is no conceptual difficulty in these steps, the actual computations are somewhat involved, due to various integer constraints and border cases. Theorem 4 below takes these complexities into account and characterizes, explicitly in terms of $k$, the parameter pair $\left(\sigma_{k}, c_{k}\right)$ of an optimal code $T_{\sigma_{k}, c_{k}}$ for $\hat{\mathcal{A}}_{k}$.

Theorem 4: Let $q=2^{-1 / k}, Q=k^{2}-\lceil k(k-1) / 4\rceil, m=$ $\left\lceil\log k^{2}\right\rceil$, and $M=\lceil\log Q\rceil$. Define the function

$\Delta(x)=2 k^{2}-2^{M+1}+x(x+1)-\frac{(k-x-2)(k-x-1)}{2}$.

Let $x_{0}$ denote the largest real root of $\Delta(x)$, and let $\xi=\left\lfloor x_{0}\right\rfloor$. Set

$$
(j, r)= \begin{cases}\left(\xi,\left\lfloor\frac{-\Delta(j)+1}{2}\right\rfloor\right), & \text { if } \Delta(\xi) \leq 2 \xi, \\ (\xi+1,0), & \text { otherwise. }\end{cases}
$$

Then, the tree $T_{\sigma_{k}, c_{k}}$, as defined by the profile (8) with $\sigma=$ $\sigma_{k}=m-M$ and

$$
c=c_{k}=k^{2}-2^{M}+\frac{j(j+1)}{2}+r,
$$

is optimal for $\hat{\mathcal{A}}_{k}$. Furthermore, $c_{k}$ is the smallest value of $c$ for any optimal tree $T_{\sigma_{k}, c}$ for $\hat{\mathcal{A}}_{k}$.

The proof of Theorem 4 is presented in Appendix B In the theorem (and its proof), we have chosen to identify the optimal tree $T_{\sigma_{k}, c}$ with the smallest possible value of $c$. It can readily be verified that this choice minimizes the variance of the code length among all optimal trees $T_{\sigma_{k}, c}$. With only minor changes in the construction and proof, one could also identify the largest value of $c$ for an optimal tree, and, thus, the full range of values of $c$ yielding optimal trees $T_{\sigma_{k}, c}$. For conciseness, we have omitted this extension of the proof.

Examples of the application of Theorem 4 are presented in Table [I]. which lists the parameters $M, j, r, \sigma_{k}, c_{k}$, and the
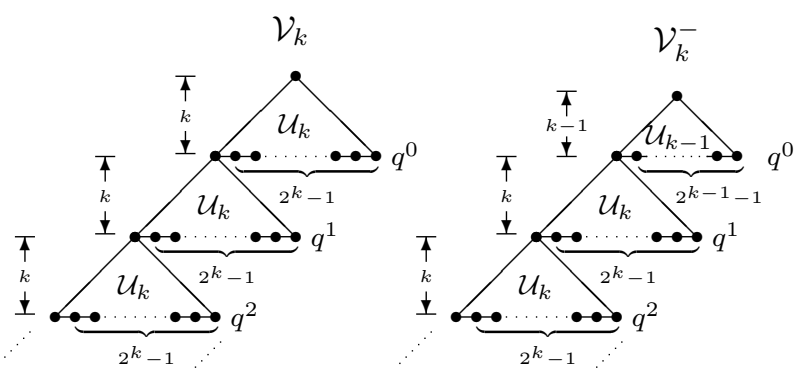

Fig. 4. Trees $\mathcal{V}_{k}$ and $\mathcal{V}_{k}^{-}$.

profile of the optimal tree $T_{\sigma_{k}, c_{k}}$ defined by the theorem, for $3 \leq k \leq 10$.

The tools derived in the proof of Theorem 4 also yield the following result, a proof of which is also presented in Appendix B

Corollary 1: Let $k>2$ and $q=2^{-1 / k}$. Then, $G_{k} \cdot G_{k}$ is not optimal for $\operatorname{TDGD}(q)$.

\section{Average code length}

The following corollary gives explicit formulas for the average code length of the codes $C_{k}$ characterized in Theorem 2 and Theorem 4. The proof is deferred to Appendix C

Corollary 2: Let $M, \Delta(x), j$, and $r$ be as defined in Theorem 4. Then, the average code length $\overline{\mathcal{L}}_{q}\left(C_{k}\right)$ for the code $C_{k}$ under $\operatorname{TDGD}(q)$, for arbitrary $q$, is given by

$$
\overline{\mathcal{L}}_{q}\left(C_{k}\right)=M+1+\frac{q^{j} V(q)}{\left(1-q^{k}\right)^{2}},
$$

where

$$
\begin{aligned}
V(q) & =1-q^{k+1}+(1-q)\left(q^{k+1}(k-j-1)+j\right) \\
& +(1-q)^{2}\left(q^{k}(2 r+\Delta(j))-r\right) .
\end{aligned}
$$

When $q=2^{-1 / k}$, we have

$$
\overline{\mathcal{L}}_{q}\left(C_{k}\right)=M+1+2 q^{j} V^{*}(q)
$$

with

$$
V^{*}(q)=1+(1-q)(q k+(2-q) j)+(1-q)^{2}(1+\Delta(j)) .
$$

\section{OPtimal CODES FOR TDGDS WiTh $q=2^{-k}$}

\section{A. The codes}

Assume $q=2^{-k}$ for some integer $k>1$. We reuse the notation $\mathcal{U}_{m}=Q_{2^{m}}$ for a uniform tree of depth $m$, assuming, additionally, that its $2^{m}$ leaves have weight one. The infinite tree (and associated multiset of leaf weights) $\mathcal{V}_{k}$ is recursively defined as follows. Start from $\mathcal{U}_{k}$, and attach to its leftmost leaf a copy of $q \mathcal{V}_{k}$. Thus, $\mathcal{V}_{k}$ has $2^{k}-1$ leaves of weight $q^{s}$ at depth $(s+1) k$ for all $s \geq 0$, and no other leaves. The related tree $\mathcal{V}_{k}^{-}$is defined by starting from $\mathcal{U}_{k-1}$, and attaching to its leftmost leaf a copy of $q \mathcal{V}_{k}$. Thus, $\mathcal{V}_{k}^{-}$has $2^{k-1}-1$ leaves of weight $q^{0}$ at depth $k-1$, and $2^{k}-1$ leaves of weight $q^{s}$ at depth $(s+1) k-1$ for all $s>0$. The trees $\mathcal{V}_{k}$ and $\mathcal{V}_{k}^{-}$are illustrated in Figure 4 
We describe a sequence of binary trees (and codes) $C_{-k}$, which, later in the section, will be shown to be optimal for TDGDs with $q=2^{-k}, k>1$. We describe the trees by layers. A layer $\mathbf{L}_{s}$ is a collection of consecutive levels of the tree, containing all the leaves with signature $s$. The structure of the layers, and how $\mathbf{L}_{s}$ unfolds into $\mathbf{L}_{s+1}$ for all $s$, are presented next, providing a full description of the trees $C_{-k}$.

Assume $k>1$ is fixed. We distinguish two main cases for the structure of $\mathbf{L}_{s}$, which depend on the value of $s$, as specified below. In the description of the layers, each tree structure is a virtual symbol. We will refer to both original and virtual symbols simply as symbols.

Case 1) $0 \leq s \leq 2^{k-1}-2$ :

Write $s=2^{i}+j-1$ with $0 \leq i \leq k-2,0 \leq j \leq 2^{i}-1$. Layer $\mathbf{L}_{s}$ consists of nodes in two levels, arranged as follows:

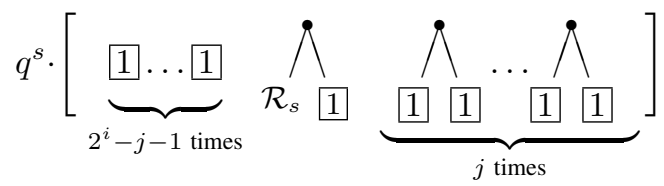

(recall that the factor $q^{s}$ multiplies all the weights of objects inside the brackets, so that the leaves denoted 1 in 22 indeed correspond to signatures $s$ ).

The symbol $\mathcal{R}_{s}$ represents a tree containing all the signatures strictly greater than $s$, scaled by $q^{-s}$. Layer $\mathbf{L}_{s}$ emerges from constructing a quasi-uniform tree for $s+2$ symbols $(s+1$ signatures $s$, and the symbol $\mathcal{R}_{s}$ ), attached to $\mathcal{R}_{s-1}$ of the previous layer if $s>0$, or to the root of the tree if $s=0$. We have $s+2=2^{i}+1+j, 0 \leq j \leq 2^{i}-1$, so the quasi-uniform tree has $2^{i}-j-1$ leaves at depth $i$, and $2 j+2$ leaves at level $i+1$, as shown in 22,

Case 2) $s \geq 2^{k-1}-1$ :

Write

$$
s=2^{k-1}-1+\left(2^{k}-1\right) \ell+j, \quad \ell \geq 0, \quad 0 \leq j<2^{k}-1 .
$$

There are five types of layers in this case, as described below. The symbol $\mathcal{R}_{s}$ in each case represents a tree containing all the signatures strictly greater than $s$ that are not contained in other virtual symbols in $\mathbf{L}_{s}$, suitably scaled by $q^{-s}$. Also, it will be convenient to use the notation $\mathcal{M}$ as shorthand for the sequence

$$
\mathcal{M}: \quad q \mathcal{V}_{k}, \underbrace{1 \ldots 1}_{2^{k}-1 \text { times }}
$$

( $\mathcal{M}$ still counts as $2^{k}$ symbols in $\mathbf{L}_{s}$ ).

(i) $0 \leq j \leq 2^{k-1}-3$ (for $k>2$ ):

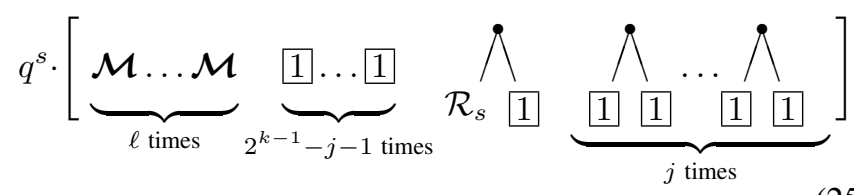

(ii) $j=2^{k-1}-2$ :

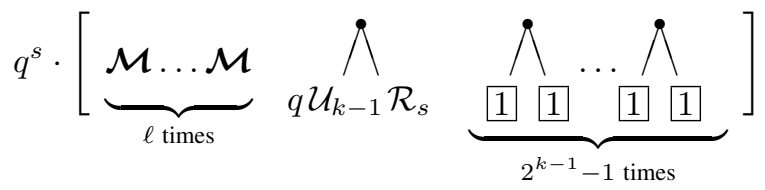

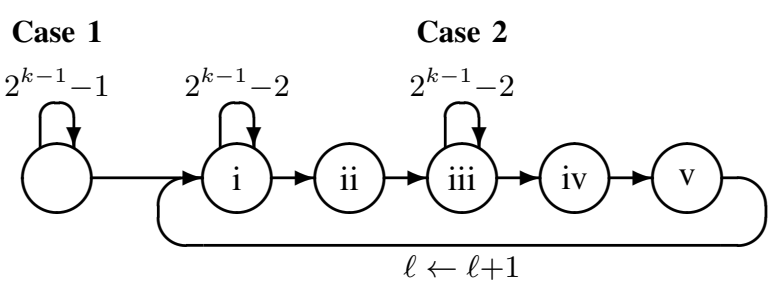

Fig. 5. Layer transitions in $C_{-k}$ for $k>2$. The expressions above the self-loops indicate the number of iterations on the given layer type before the transition to the next type.

(iii) $2^{k-1}-1 \leq j \leq 2^{k}-4$ :

$$
q^{s} \cdot[\underbrace{\mathcal{M} \ldots \mathcal{M}}_{\ell \text { times }} \underbrace{11 \ldots 1}_{3 \cdot 2^{k-1}-2-j \text { times }} \bigwedge_{\mathcal{U}_{k-1}} \mathcal{R}_{s} \underbrace{\bigwedge_{1}^{1} \bigwedge_{1}^{1}}_{j-2^{k-1}+1 \text { times }}]
$$

(iv) $j=2^{k}-3$ :

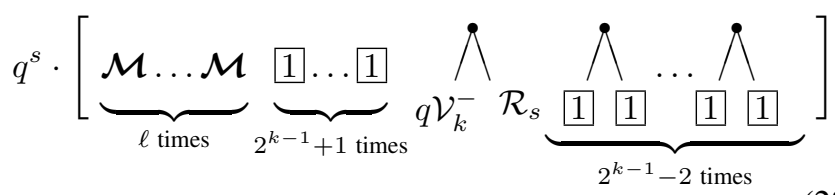

(v) $j=2^{k}-2$ :

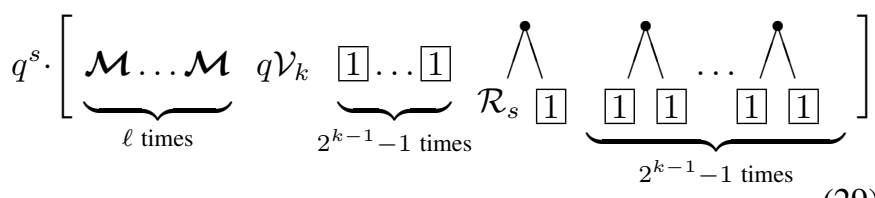

The last layer from Case 1 contains all the signatures $s^{\prime}=2^{k-1}-2$. All signatures $s>s^{\prime}$ are contained in $\mathcal{R}_{s^{\prime}}$. In particular, there are $2^{k-1}$ signatures $s^{\prime}+1=2^{k-1}-1$. Assume $k>2$. A quasi-uniform tree with $2^{k-1}+1$ leaves is constructed, rooted at $\mathcal{R}_{s^{\prime}}$. This tree has $2^{k-1}-1$ leaves labeled $s^{\prime}+1$ at depth $k-1$ from its root, and two leaves at depth $k$, one of which is labeled $s^{\prime}+1$, and one that serves as the root for $\mathcal{R}_{s^{\prime}+1}$. This is consistent with the structure of the first layer in Case 2 shown in 25, with $s=s^{\prime}+1, \ell=0$ and $j=0$. From that layer on, layers of types (i) $-(\mathrm{v})$ above unfold following the cyclic pattern shown in Figure 5. Layers of types (i) and (iii) are repeated $2^{k-1}-2$ times each in the cycle, which is closed by a transition from a layer of type (v) back to one of type (i), corresponding to an increment of the value of $\ell$ by one.

When $k=2$, layers of type (i) or (iii) are not used. In this case, the only layer in Case 1 contains the signature 0 . A uniform tree $\mathcal{U}_{2}$ is constructed, rooted at $\mathcal{R}_{0}$. One pair of sibling leaves is assigned to signature 1 , while the other pair is assigned to $\mathcal{R}_{1}$ and $\mathcal{U}_{1}$, attaining a configuration of type (ii) in Case 2. From that point on, the cyclic layer sequence is (ii) $\rightarrow$ (iv) $\rightarrow$ (v) $\rightarrow$ (ii).

The fine details of the various layer transitions, justifying the structure in Figure 5, are given in Appendix D. The structure 


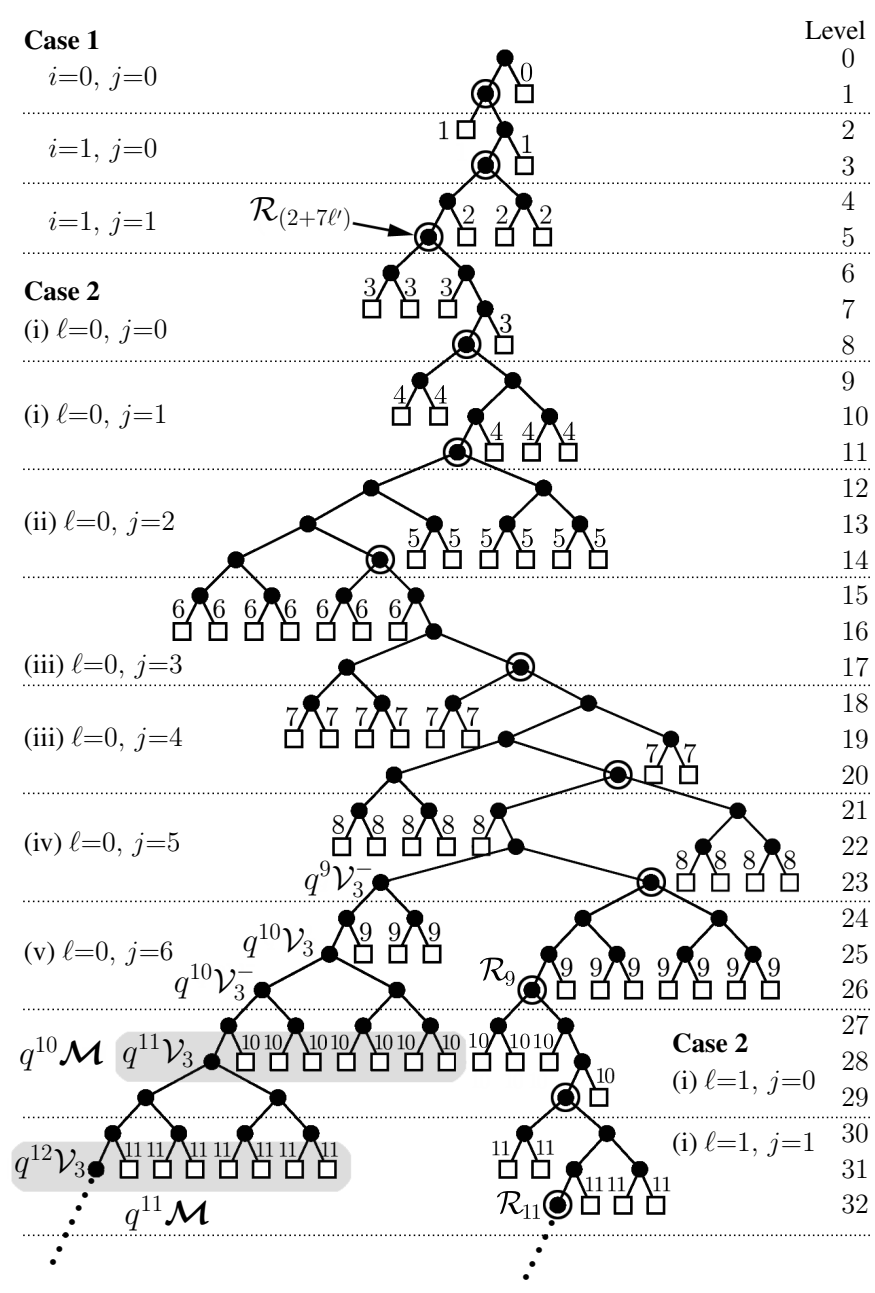

Fig. 6. Top levels comprising layers $\mathbf{L}_{s}$ for $s \leq 11$ in the optimal tree $C_{-3}\left(q=\frac{1}{8}\right)$. Leaf signatures are noted; dotted lines separate layers $\mathbf{L}_{s}$, and circled nodes represent roots of trees $\mathcal{R}_{s}$. Grayed ovals represent sequences $q^{s} \mathcal{M}$.

is also illustrated by the example in Figure 6 which shows the layers $\mathbf{L}_{s}$ for $s \leq 11$ in $C_{-3}$.

Due to the cyclic nature of the construction, the subtree $\mathcal{R}_{s}, s \geq 2^{k-1}-2$ is, in general, identical to all subtrees $\mathcal{R}_{s+\left(2^{k}-1\right) \ell^{\prime}}, \ell^{\prime} \geq 0$, up to appropriate scaling by $q^{\left(2^{k}-1\right) \ell^{\prime}}$. In the example of Figure 6 , the tree $\mathcal{R}_{9}$ is identical to the tree $\mathcal{R}_{2}$, indicated in the figure as $\mathcal{R}_{2+7 \ell^{\prime}}$. An additional source of selfsimilarity is provided by the trees $\mathcal{V}_{k}$ and $\mathcal{V}_{k}^{-}$; in Figure 6, the sub-tree labeled $q^{10} \mathcal{V}_{3}^{-}$is identical to that labeled $q^{9} \mathcal{V}_{3}^{-}$, etc. Overall, although the width of the tree is unbounded (driven by the $\ell$ copies of $\mathcal{M}$ in each layer of Case 2), the total number of distinct sub-trees in $C_{-k}$ is finite.

The following theorem enumerates the code lengths assigned to signatures by the codes $C_{-k}$. It follows immediately from the description of the codes in 22) and 25 - 29.

Theorem 5: Code $C_{-k}, k>1$, assigns code lengths $\Lambda_{s}$ or $\Lambda_{s}+1$ to signatures $s$ according to the expressions for $\Lambda_{s}$ and the codeword counts in Tables $\mathrm{III}$ and IV] corresponding, respectively, to the cases $0 \leq s \leq 2^{k-1}-2$ (Case 1) and $s \geq 2^{k-1}-1$ (Case 2).

We now present some auxiliary results that will be useful
TABLE III

CODE LENGTHS AND CODEWORD COUNTS FOR CODES $C_{-k}$ ON SIGNATURES $s, 0 \leq s \leq 2^{k-1}-2$.

\begin{tabular}{|l|c|c|}
\hline Case 1: & $0 \leq s \leq 2^{k-1}-2, \quad s=2^{i}+j-1, \quad 0 \leq i \leq k-2$ \\
& $\Lambda_{s}=(s+2)(i+1)-2^{i+1}$ & \\
\hline \multirow{3}{*}{ Range of $j$} & \multicolumn{2}{|c|}{ Number of codewords (signatures) } \\
\hline $0 \leq j \leq 2^{i}-1$ & length $\Lambda_{s}$ & length $\Lambda_{s}+1$ \\
\hline $0 y$ & $\left(2^{i}-j-1\right)$ & $2 j+1$ \\
\hline
\end{tabular}

TABLE IV

CODE LENGTHS AND CODEWORD COUNTS FOR CODES $C_{-k}$ ON SIGNATURES $s \geq 2^{k-1}-1$.

\begin{tabular}{|c|c|c|}
\hline $\begin{array}{ll}\text { Case 2: } & s \geq 2^{k-1} \\
& \Lambda_{s}=(s+\end{array}$ & $\begin{array}{l}s=2^{k-1}-1+\left(2^{k}-1\right) \\
-2^{k}\end{array}$ & $\ell \geq 0$ \\
\hline \multirow[b]{2}{*}{ Range of $j$} & \multicolumn{2}{|c|}{ Number of codewords (signatures) } \\
\hline & length $\Lambda_{s}$ & length $\Lambda_{s}+1$ \\
\hline \multirow{5}{*}{$\begin{array}{l}0 \leq j \leq 2^{k-1}-3 \\
j=2^{k-1}-2 \\
2^{k-1}-1 \leq j \leq 2^{k}-4 \\
j=2^{k}-3\end{array}$} & $\left(2^{k}-1\right) \ell+\left(2^{k-1}-j-1\right)$ & $2^{j}+1$ \\
\hline & $\left(2^{k}-1\right) \ell$ & $2^{k}-2$ \\
\hline & $\left(2^{k}-1\right) \ell+3 \cdot 2^{k-1}-2-j$ & $2 j+2-2^{k}$ \\
\hline & $\left(2^{k}-1\right) \ell+2^{k-1}+1$ & $2^{k}-4$ \\
\hline & $\left(2^{k}-1\right) \ell+2^{k-1}-1$ & $2^{k}-1$ \\
\hline
\end{tabular}

in proving the optimality of the codes $C_{-k}$. We rely on the following relations, which are readily derived from the definitions of the respective trees, under the assumption $q=2^{-k}$ :

$$
w\left(\mathcal{U}_{k}\right)=2 w\left(\mathcal{U}_{k-1}\right)=w\left(\mathcal{V}_{k}\right)=2 w\left(\mathcal{V}_{k}^{-}\right)=q^{-1}
$$

The next lemma bounds the weight of the symbol $\mathcal{R}_{s}$ in 22 and 25]-29].

Lemma 11: When $s \leq 2^{k-1}-2$ (Case 1), we have $0 \leq$ $w\left(\mathcal{R}_{s}\right) \leq \frac{7}{9}$. When $s>2^{k-1}-2$ (Case 2$)$, we have $\frac{1}{2} \leq$ $w\left(\mathcal{R}_{s}\right) \leq 1$.

Proof: For $s \leq 2^{k-1}-2$, we have

$$
\begin{aligned}
w\left(\mathcal{R}_{s}\right) & =\sum_{s^{\prime}=s+1}^{\infty}\left(s^{\prime}+1\right) q^{-s} w\left(s^{\prime}\right) \\
& =\sum_{r=0}^{\infty}(s+r+2) q^{r+1}=\frac{(s+1)(1-q)+1}{(1-q)^{2}} q .
\end{aligned}
$$

The right-hand side of (31) increases with $s$. Setting $s=$ $2^{k-1}-2=\frac{1}{2 q}-2$, we obtain $w\left(\mathcal{R}_{s}\right)=\frac{1}{2}\left(1+\frac{q(1+q)}{(1-q)^{2}}\right)$, which satisfies the claimed upper bound for $q \leq \frac{1}{4}$. When $s \geq 2^{k-1}-1, \mathcal{R}_{s}$ contains all the signatures $s^{\prime}>s$ (with their weights scaled by $q^{-s}$ ) that are not contained in the components $q \mathcal{V}_{k}$ of the groups $\mathcal{M}$, or in a possible sibling $q \mathcal{U}_{k-1}$ or $q \mathcal{V}_{k}^{-}$of $\mathcal{R}_{s}$. Write $s$ as in 23. The scaled total weight of signatures $s^{\prime}>s$ is

$$
\begin{aligned}
W_{s} & =q^{-s} \sum_{r=0}^{\infty}(s+2+r) q^{s+1+r}=\frac{(s+2) q}{1-q}+\frac{q^{2}}{(1-q)^{2}} \\
& =\frac{2 q(1+j)+1}{2(1-q)}+\frac{q^{2}}{(1-q)^{2}}+\ell,
\end{aligned}
$$

where the last equality follows by applying 23) and substituting $q^{-1}$ for $2^{k}$. Let $W_{s}^{\prime}$ denote the part of $W_{s}$ that is contained in the symbols $q \mathcal{V}_{k}, q \mathcal{U}_{k-1}$, or $q \mathcal{V}_{k}^{-}$mentioned above. Observing the layer structures in (25)-(29), and applying (30), 
we obtain $W_{s}^{\prime}=\ell+\delta$, where:

$$
\delta= \begin{cases}0, & 0 \leq j \leq 2^{k-1}-3 \\ \frac{1}{2}, & 2^{k-1}-2 \leq j \leq 2^{k}-3 \\ 1, & j=2^{k}-2\end{cases}
$$

The claim of the lemma for $s>2^{k-1}-2$ follows by writing $w\left(\mathcal{R}_{s}\right)=W_{s}-W_{s}^{\prime}$, observing that $w\left(\mathcal{R}_{s}\right)$ increases monotonically with $j$, and bounding $w\left(\mathcal{R}_{s}\right)$, as an elementary function of $q$, in the interval $0<q \leq \frac{1}{4}$ for each of the cases in 32. Notice that due to the mentioned monotonicity, $w\left(\mathcal{R}_{s}\right)$ is evaluated only at the ends of the ranges of $j$ in (32), and we substitute $q^{-1}$ for $2^{k}$.

The following is an immediate consequence of Lemma 11 .

Corollary 3: Let $\mathcal{R}_{s}^{\prime}$ denote the virtual symbol containing $\mathcal{R}_{s}$ in each layer $\mathbf{L}_{s}$ listed in 22 and (25)-29]. Then, after scaling by $q^{-s}$, all the symbols to the left of $\mathcal{R}_{s}^{\prime}$ in $\mathbf{L}_{s}$ are of weight 1 , all the symbols to its right are of weight 2 , and we have $1 \leq w\left(\mathcal{R}_{s}^{\prime}\right) \leq 2$.

Proof: The claims on the symbols to the left and to the right of $\mathcal{R}_{s}^{\prime}$ follow from 30 and the definition of the notation $\mathcal{M}$ in 24. As for $\mathcal{R}_{s}^{\prime}$, we have $w\left(\mathcal{R}_{s}^{\prime}\right)=1+w\left(\mathcal{R}_{s}\right)$, and the claim of the corollary follows by applying Lemma 11

Theorem 6: The prefix code $C_{-k}$ is optimal for $\operatorname{TDGD}(q)$ with $q=2^{-k}, k>1$.

Proof: As before, we rely on the method from [2]. The reduced sources are defined by $\mathcal{S}_{s}=\mathcal{H}_{s} \cup \mathcal{F}_{s}$, where $\mathcal{H}_{s}$ denotes, as before, the multiset of signatures strictly smaller than $s$, and the multiset $\mathcal{F}_{s}$ is essentially identical to the layer $\mathbf{L}_{s}$ defined in 22) and 25]-29. The steps taking a reduced source to one of lower order follow the layer "unfolding" steps listed in the description of the codes $C_{-k}$ (see the discussion following (22) and (25)-(29), and Appendix D), in reverse order (bottom-up). It remains to show that these steps correspond to a valid sequence of mergers in the Huffman procedure. Consider a layer $\mathbf{L}_{s}$, and let $\psi_{1}, \psi_{2}, \ldots, \psi_{N}$ denote its symbols, listed from left to right, as shown in (22) and (25) 29]. It is readily verified that $N=2^{i}$ for a layer (22), with $i$ as defined in Case 1 , and that $N$ is divisible by $2^{k-1}$ in layers of type (i)-(ii), and by $2^{k}$ in layers of type (iii)-(v). By Corollary 3, the $\psi_{j}$ are ordered by increasing weight order, and, since $q<1 / 2$, the weight of any $\psi_{j}$ is smaller than any weight in $\mathcal{H}_{s}$. Thus, the Huffman procedure on $\mathcal{S}_{s}$ starts by pairing symbols in $\mathbf{L}_{s}$. Now, it also follows from Corollary 3 that the merger of any two of the $\psi_{j}$ results in a combined weight that is at least as large as any weight in the layer. Thus, merging $\psi_{2 j-1}$ with $\psi_{2 j}, 1 \leq j \leq N / 2$, is a valid sequence of steps in the Huffman procedure on $\mathbf{L}_{s}$. Moreover, since there is at most one symbol of weight different from 1 or 2 (after scaling), and strictly between them, the resulting sequence of merged weights includes weights $2, \omega$, and 4 , with $2 \leq \omega \leq 4$, with at most one symbol of weight $\omega$. We iterate the argument until the signatures $s-1$ get incorporated, and $\mathbf{L}_{s-1}$ gets formed (see Appendix $\mathrm{D}$, reaching, thus, the reduced source $\mathcal{S}_{s-1}$. Proceeding recursively, we reach the reduced source $\mathcal{S}_{0}$, which coincides with the layer $\mathbf{L}_{0}$. As described in 22) for $s=0$, this layer consists of one virtual symbol formed by $\mathcal{R}_{0}$ and the symbol 0 joined under the root

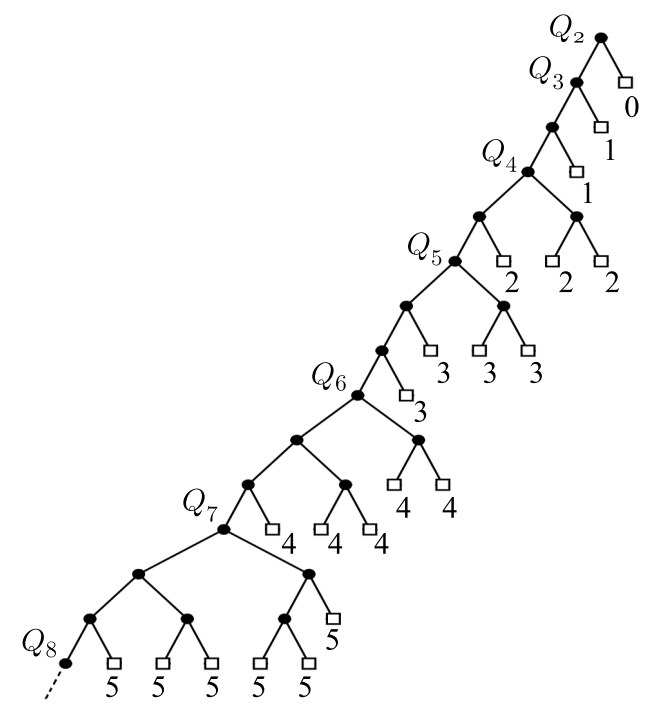

Fig. 7. Top of the limit tree $C_{-\infty}$.

of the tree $C_{-k}$ (thus, the Huffman procedure on $\mathcal{S}_{0}$ is trivial in this case).

\section{B. A limit code}

The sequence of optimal codes $C_{-k}$ stabilizes in the limit of $k \rightarrow \infty(q \rightarrow 0)$, as stated in the following corollary.

Corollary 4: When $k \rightarrow \infty$, the sequence of optimal trees $C_{-k}$ converges to a limit tree $C_{-\infty}$ that can be constructed as follows: start with $Q_{n}$ for $n=2$, recursively replace the leftmost leaf of the deepest level of the current tree by $Q_{n+1}$, and increase $n$.

Proof: The corollary is proved by observing that the part of the tree corresponding to $0 \leq s \leq 2^{k-1}$ in Theorem 6 remains invariant for all $k^{\prime} \geq k$. This corresponds to the layers $\mathbf{L}_{s}$ of Case 1.

The limiting property of $C_{-\infty}$ in connection with the TDGD is mentioned also in [11, Ch. 5]. Figure 7 shows the first fourteen levels of $C_{-\infty}$. Notice that the first eleven levels coincide with those of $\mathrm{C}_{-3}$ in Figure 6, up to reordering of nodes at each level. Explicit encoding with $C_{-\infty}$ can be done as follows. Given a pair $(i, j)$, with signature $s=i+j$, we write $s=2^{t}-1+r$, with $0 \leq r \leq 2^{t}-1$ and $t \geq 0$. We encode $(i, j)$ with a binary codeword $x y$, where $x=1^{\overline{(t}-1)(s+1)+2 r+1}$ identifies the path to the root of the quasi-uniform tree that contains all the leaves of signature $s$, and $y=Q_{s+2}(i+1)$. The resulting code length distribution for signature $s$ is: $2^{t}-1-r$ signatures encoded with length $(t-1)(s+2)+2 r+2,2 r+1$ signatures encoded with length $(t-1)(s+2)+2 r+3$.

The following corollary shows the average code length attained by $C_{-\infty}$ on an arbitrary TDGD.

Corollary 5: The average code length of the limit code $C_{-\infty}$ under $\operatorname{TDGD}(q)$ is given by

$$
\overline{\mathcal{L}}_{q}\left(C_{-\infty}\right)=1+\frac{1}{1-q} \sum_{t \geq 0} q^{2^{t}}\left(2^{t}(1-q)+2\right) .
$$

Proof: For $s \geq 0$, let $r$ and $t, t \geq 0,0 \leq r \leq 2^{t}-1$, be the (uniquely determined) integers such that $s=2^{t}-1+r$. 
By Corollary 4 and the ensuing discussion, we can write

$$
\overline{\mathcal{L}}_{q}\left(C_{-\infty}\right)=(1-q)^{2} \sum_{t \geq 0} \sum_{s=2^{t}-1}^{2^{t+1}-2} q^{s} D(t, s),
$$

where

$$
D(t, s)=((t-1)(s+2)+2 r+2)(s+1)+2 r+1 .
$$

Substituting $r=s-2^{t}+1$ and carrying out the inner summation in (33), we obtain

$$
\overline{\mathcal{L}}_{q}\left(C_{-\infty}\right)=(1-q)^{2} \sum_{t \geq 0}\left(q^{2^{t+1}-1} A(t)+q^{2^{t}-1} B(t)\right),
$$

for some functions $A(t)$ and $B(t)$. It can be verified by symbolic manipulation that

$$
B(0)=\frac{1-q^{2}+2 q}{(1-q)^{3}}
$$

and

$$
A(t-1)+B(t)=q \frac{2^{t}-2^{t} q+2}{(1-q)^{3}} .
$$

Substituting in 34, after rearranging terms, we obtain

$$
\begin{aligned}
\overline{\mathcal{L}}_{q}\left(C_{-\infty}\right) & =(1-q)^{2}\left(B(0)+\sum_{t \geq 1} q^{2^{t}-1}(A(t-1)+B(t))\right) \\
& =(1-q)^{2}\left(\frac{1-q^{2}+2 q}{(1-q)^{3}}+\sum_{t \geq 1} q^{2^{t}} \frac{2^{t}-2^{t} q+2}{(1-q)^{3}}\right) \\
& =1+\frac{1}{1-q} \sum_{t \geq 0} q^{2^{t}}\left(2^{t}(1-q)+2\right) .
\end{aligned}
$$

\section{PRactical Considerations AND Redundancy}

In a practical situation, one could use the codes $C_{k}$ for $q \geq \frac{1}{2}$, and the codes $C_{-k}$ for $q<\frac{1}{2}$. However, a lower complexity alternative, which incurs a modest code length penalty (as shown in Figure 8), is to use $C_{-\infty}$ in lieu of the codes $C_{-k}$, up to the value of $q$ where switching to $C_{1}$ gives better average code length. The crossover point is at $q \approx 0.33715$.

Encoding a symbol pair $(x, y)$ with a code $C_{k}$ is of about the same complexity as two encodings of individual symbols with a Golomb code of order $k$. As described in Theorem 2, the encoding with $C_{k}$ entails unary encodings of $\lfloor x / k\rfloor$ and $\lfloor y / k\rfloor$, which would also be needed with the Golomb code. Given the profile of the top code $T_{k}=T_{\sigma_{k}, c_{k}}$, determined in Theorem 4 . encoding with $T_{k}$ requires comparing the index of the pair $(x \bmod k, y \bmod k)$ with at most two fixed thresholds, to determine the corresponding code length (which can assume up to three consecutive integer values). The codeword is then computed directly from the index. Each encoding with the Golomb code, on the other hand, requires one comparison with a fixed threshold to determine the code length of each $Q_{k}$ component, or a total of two for the pair $(x, y)$.

As in the one-dimensional case (see, e.g., [3], [15]), when encoding a sequence $x_{1}, x_{2}, \ldots, x_{2 t}, \ldots$, the best code for the next pair $\left(x_{2 t-1}, x_{2 t}\right)$ can be determined adaptively, driven by the sufficient statistic $S_{t}=t^{-1} \sum_{j=1}^{2 t-2} x_{j}$. The crossover points for the estimates of the code parameter $k$ can be precomputed and stored in terms of the statistic $S_{t}$. The onedimensional code has a slight advantage in the adaptation, in that it can adapt its statistic with every symbol, whereas the two-dimensional code can only do it every two symbols. Depending on the application, this advantage is likely to be superseded by the redundancy advantage of the two-dimensional code. Also as in the one-dimensional case, there are certain complexity advantages, in both encoding and adaptation when using the subset of parameters of the form $k=2^{r}$. In this case, an adaptation strategy that estimates the best parameter $r$ directly from the statistic $S_{t}$, without the need to compare it with precomputed crossover points, can be derived for the codes $C_{k}$, as was done in [3] and [15] for two-sided geometric distributions. We omit the details, since both the technique and the resulting parameter estimation method are similar to those in the references.

Figure 8 presents plots of redundancy for various code families as a function of $q$, measured in bits per integer symbol relative to the entropy of the geometric distribution (recall that the latter is given by $H(q)=\frac{h(q)}{1-q}$, where $h(q)$ is the binary entropy function [2]). Plots are shown for the optimal prefix code for each value of $q$ (estimated numerically over a dense grid of values of $q$, and in sufficient precision to make the estimation error smaller than the plot resolution), the best Golomb code, the best code $C_{-k}$ or $C_{k}$ for each $q$, and the limit code $C_{-\infty}$. Here, "the best Golomb code" means the code $G_{k}$ that minimizes (over $k$ ) the code length for the given value of $q$; similar minimizations are used for the best codes $C_{-k}$ and $C_{k}$ for each $q$. In the figure, we can observe the advantage in redundancy for the codes $C_{-k}$ (or $C_{-\infty}$ ) and $C_{k}$ over Golomb codes, except in the region where the best codes of both types are equivalent (i.e., the optimality regions of $C_{1}$ and $C_{2}$ ). The redundancy advantage is near $2: 1$ (as expected) at the limit of $q \rightarrow 0$ and it peaks near $q=0.28$ (at more than $13.6: 1$ ). A redundancy advantage close to $2: 1$ is observed also as $q \rightarrow 1$. The advantage of $C_{k}$ over symbol-bysymbol Golomb codes is consistent with Corollary 1, and, in fact, the plot in Figure 8 can be regarded as "visual evidence" for the corollary. Figure 9 plots the corresponding curves for the relative redundancy, i.e., the redundancy normalized by the per-symbol entropy $H(q)$ for each plotted value of $q$. We observe that although the relative redundancy for all the codes considered converges to zero, as expected, when $q \rightarrow 1$ (since $H(q) \rightarrow \infty$ ), the decay is very slow for most of the interval, and the curves fall to zero "suddenly", with infinite slope, near $q=1$. This is due to the slow rate of growth of $H(q)$, which behaves asymptotically as $-\log (1-q)$ near the limit point.

It is apparent from Figure 8 that as the redundancy of the codes $C_{k}$ peaks in the transitions between one "best" value of $k$ and the next, the estimated redundancy of the optimal codes remains rather flat. This poses the question, which also remains open, of whether other sequences of codes with simple descriptions and encoding/decoding procedures could be found, that would more closely track the redundancy curve of the optimal codes. 

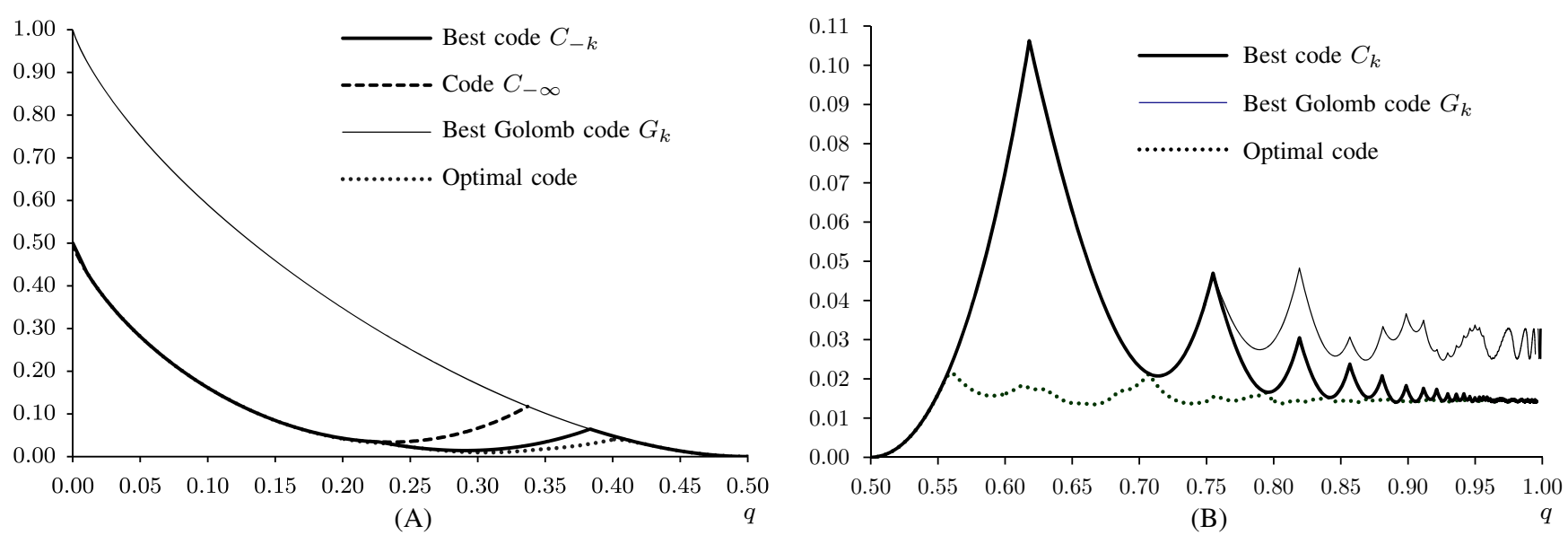

Fig. 8. Redundancy (in bits/integer symbol) for the optimal prefix code (estimated numerically), the best Golomb code, the limit code $C_{-\infty}$, and the best code $C_{-k}$ or $C_{k}$ for each value of $q$, (A) $0<q<\frac{1}{2}$, (B) $\frac{1}{2} \leq q<1$. The limit code $C_{-\infty}$ is plotted up to $q=0.33715 \ldots$, where its curve intersects that of $C_{1}$ (or, equivalently, $C_{-1}$ ).

The asymptotic behavior of the redundancy of $C_{k}$ in the regime $q \rightarrow 1$, shown in more detail in Figure 10, is oscillatory, as is also the case for Golomb codes [2]. The limiting behavior of the redundancy can be characterized precisely, as we show next.

Corollary 6: Let $\lambda_{k}=2^{M} / k^{2}$, where $M$ is as defined in Theorem 4. As $k \rightarrow \infty$, the redundancy of the code $C_{k}$ at $q=2^{-1 / k}$ is

$$
\begin{aligned}
R(k)= & \frac{1}{2}\left(1+\log \lambda_{k}\right)+2^{1-2 \sqrt{\lambda_{k}-\frac{1}{2}}}\left(1+\frac{2}{\log e} \sqrt{\lambda_{k}-\frac{1}{2}}\right) \\
& -\log (e \log e)+o(1) .
\end{aligned}
$$

Remark. We have $\frac{3}{4} \lesssim \lambda_{k} \lesssim \frac{3}{2}$, where $\lesssim$ denotes inequality up to asymptotically negligible terms. For large $k$, as $k$ increases, $\lambda_{k}$ sweeps its range decreasing from $\frac{3}{2}$ to $\frac{3}{4}$, at which point $M_{k}$ increases by one, and $\lambda_{k}$ resets to $\frac{3}{2}$, starting a new cycle.

Proof of Corollary 65. We derive, from 21], an asymptotic expression for the code length $\overline{\mathcal{L}}_{q}\left(C_{k}\right)$. To estimate the parameter $j$ in (21), we need to solve the quadratic equation $\Delta(x)=0$, with $\Delta(x)$ as defined in Theorem 4. Writing $2^{M}=\lambda_{k} k^{2}$, it is readily verified that the largest solution to the equation is $\xi=\left(2 \sqrt{\lambda_{k}-\frac{1}{2}}-1\right) k+O(1) \triangleq \alpha k+O(1)$. Thus, $j=\alpha k+O(1)$, and $q^{j}=2^{-\alpha}+O\left(k^{-1}\right)$. Writing also $q=2^{-1 / k}=1-\frac{\ln 2}{k}+O\left(k^{-2}\right)$, and noting that $\Delta(j)=O(k)$, we obtain, from (21),

$$
\overline{\mathcal{L}}_{q}\left(C_{k}\right)=M+1+2^{1-\alpha}(1+(1+\alpha) \ln 2)+o(1) .
$$

As for the entropy, we have

$$
\begin{aligned}
H(q) & =\frac{-q \log q}{1-q}-\log (1-q)=\log (e \log e)+\log k+o(1) \\
& =\log (e \log e)+\frac{1}{2}\left(M-\log \lambda_{k}\right)+o(1) .
\end{aligned}
$$

The claimed result $(35)$ follows by substituting the asymptotic expressions for $\overline{\mathcal{L}}_{q}\left(C_{k}\right)$ and $H(q)$ in the formula for the redundancy per symbol, namely, $R(k)=\frac{1}{2} \overline{\mathcal{L}}_{q}\left(C_{k}\right)-H(q)$.

The limits of oscillation of the function $R_{k}$ can be obtained by numerical computation, yielding $R_{1} \triangleq \liminf _{k \rightarrow \infty} R(k)=$ $0.014159 \ldots$ and $R_{2} \triangleq \limsup _{k \rightarrow \infty} R(k)=0.014583 \ldots$. These limits are shown in Figure 10 The corresponding limits for the redundancy of the Golomb codes are, respectively, $R_{1}^{\prime}=0.025101 \ldots$ and $R_{2}^{\prime}=0.032734 \ldots$ [2].

Corollary 6 applies to the discrete sequence of redundancy values at the points $q=2^{-1 / k}$. It is not difficult to prove that the same behavior, and in particular the limits $R_{1}$ and $R_{2}$, apply also to the continuous redundancy curve obtained when using the best code $C_{k}$ at each arbitrary value of $q$. This follows from the readily verifiable fact that as $q$ varies in the interval $2^{-1 / k} \leq q \leq 2^{-1 /(k+1)}$, the maximal variation in both the code length under $C_{k}$ and the distribution entropy is bounded by $O\left(k^{-1}\right)$. Figure 10 suggests that the same oscillatory behavior might apply also to the redundancy curve of the optimal prefix code for each value of $q$. It follows from the foregoing discussion that this is true for the limit superior $R_{2}$. The question remains open, however, for the limit inferior $R_{1}$, which is an upper bound for the limit inferior of the optimal redundancy.

\section{APPENDIX A \\ ProOfS FOR SUbSECTION IV-B}

We recall that we consider a 4-uniform probability distribution $\mathbf{p}=\left(p_{1}, p_{2}, \ldots, p_{N}\right)$, where probabilities are listed in non-increasing order, and an optimal tree $T$ for $\mathbf{p}$, with $f_{T} \leq 2$. We define $m=\lceil\log N\rceil$, and we denote by $n_{\ell}$ the number of leaves at depth $\ell$ in $T$.

Proof of Lemma 6. Say $T$ has $t>0$ leaves at depths $\ell<$ $m-2$. Then, $T$ has no leaves at depths $\ell^{\prime} \geq m$, and it can have a total of at most $2^{m-1}-3 t$ leaves altogether. But $N>2^{m-1}$, a contradiction. Say now that $T$ has nodes at depth $m+2$. Then all of its leaves must be at depths $\ell^{\prime} \geq m$, and some must be at depths strictly greater than $m$. Thus, $T$, being full, must have more than $2^{m} \geq N$ leaves, again a contradiction. The 

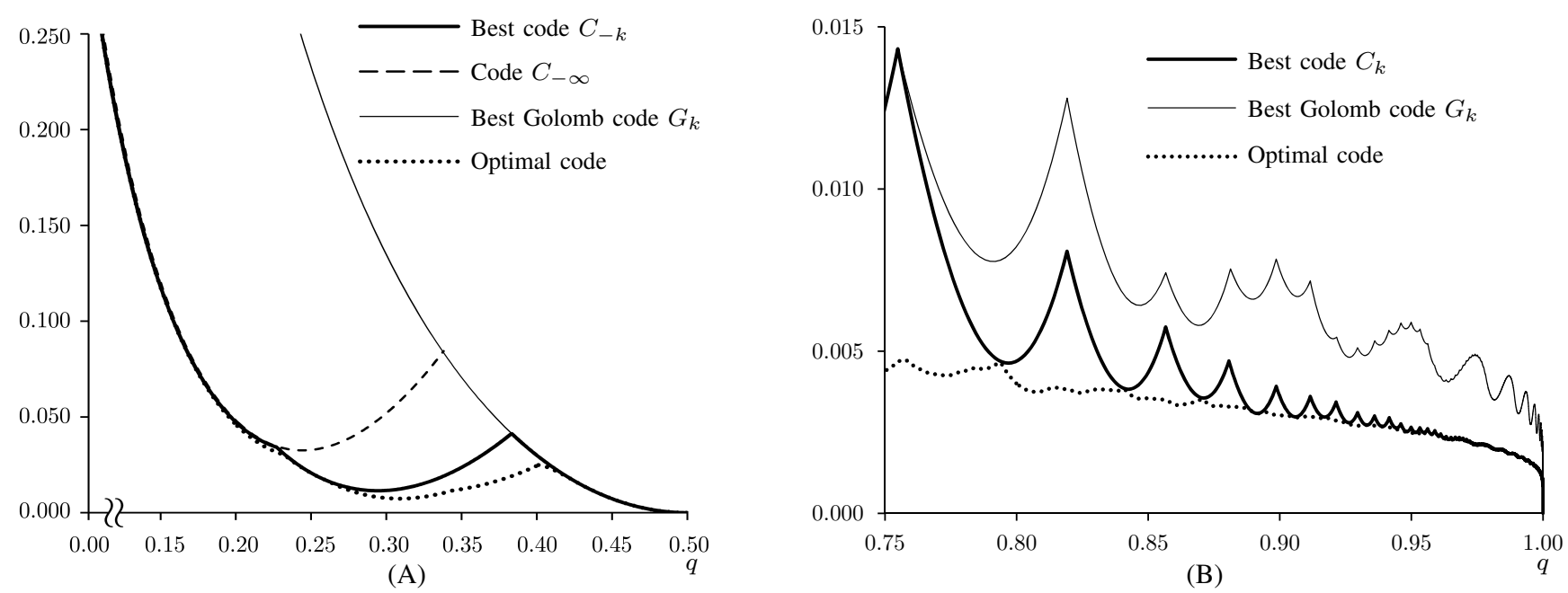

Fig. 9. Relative redundancy (redundancy normalized by the per-symbol entropy) for the codes of Figure 8 The interval $0.5 \leq q<0.75$ is omitted from (B), as the best codes $C_{k}$ and $G_{k}$ coincide over that interval.

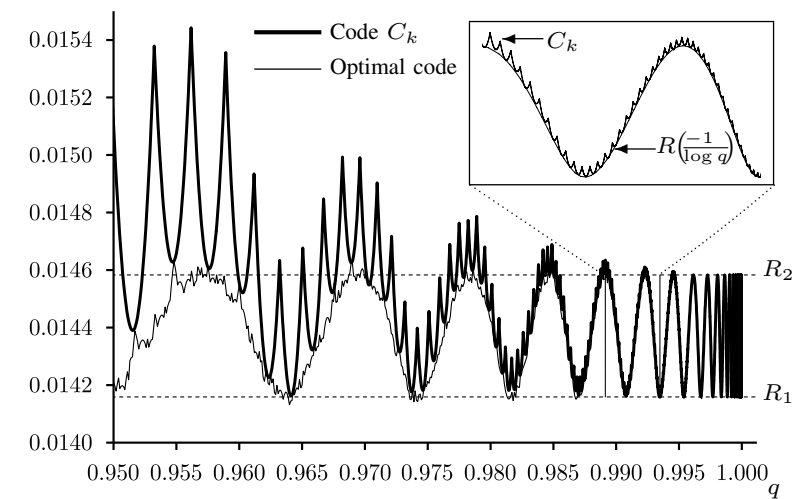

Fig. 10. Redundancy as $q \rightarrow 1(k \rightarrow \infty)$. Dashed lines show the asymptotic limits $R_{1}$ and $R_{2}$. The inset closes up further on a narrow segment, showing the redundancy of the codes $C_{k}$ vs. the asymptotic estimate 35 .

second claim of the lemma is a straightforward consequence of $f_{T} \leq 2$.

Proof of Lemma 7. Let $\mathbf{N}_{T}=\left(n_{M-1}, n_{M}, n_{M+1}\right)$ be the compact profile of a tree $T$ with $N$ leaves and $f_{T} \leq 2$. Clearly, $n_{M+1}$ must be even, and we write $n_{M+1}=2 c$ for some nonnegative integer $c$. The components of $\mathbf{N}_{T}$ must satisfy

$$
n_{M-1}+n_{M}+2 c=N \text {. }
$$

By Kraft's equality, which must hold for the full tree $T$, we have

$$
4 n_{M-1}+2 n_{M}+2 c=2^{M+1},
$$

which holds also in the case $c=0$. From (36) and (37), we obtain

$$
n_{M-1}=2^{M}-N+c .
$$

Now, from (38) and (36), we obtain

$$
n_{M}=2 N-2^{M}-3 c .
$$

Equations (38) and (39), together with the definition of $c$ yield the profile (8). The valid range of variation of $c$ is determined by the non-negativity constraints on the entries of the profile. When $M=m-1(\sigma=1)$, the lower limit $\underline{c}_{\sigma}=N-2^{m-1}$ is determined by the nonnegativity of $n_{M-1}$. Since $2^{M} \geq N$ when $M=m$, the lower limit is the trivial $\underline{c}_{0}=0$ in this case. In both cases, the upper limit $\bar{c}_{\sigma}=\left\lceil\frac{2 N-2^{M}}{3}\right\rceil$ is determined by the nonnegativity of $n_{M}$.

Proof of Lemma 8. For a given value of $\sigma \in\{0,1\}$, assume $c$ and $c^{\prime}$ are indices such that $\underline{c}_{\sigma}<c^{\prime} \leq c \leq \bar{c}_{\sigma}$, and let $\mathbf{s}_{\sigma}$ be the segment of $\mathbf{s}$ corresponding to $\sigma$. By (10) and the monotonicity of the weights, we have

$$
\begin{aligned}
D_{\sigma, c^{\prime}} & =p_{N-2 c^{\prime}+1}+p_{N-2 c^{\prime}+2}-p_{2^{M}-N+c^{\prime}} \\
& \leq p_{N-2 c+1}+p_{N-2 c+2}-p_{2^{M}-N+c}=D_{\sigma, c} .
\end{aligned}
$$

Thus, if $D_{\sigma, c}<0$ then $D_{\sigma, c^{\prime}}<0$, and if $D_{\sigma, c}=0$ then $D_{\sigma, c^{\prime}} \leq 0$. It follows that $\mathbf{s}_{\sigma}$ is non-decreasing. It remains to prove that $-\operatorname{sg}\left(D_{1, \underline{c}_{1}+1}\right) \leq \operatorname{sg}\left(D_{0,1}\right)$. Assume that $D_{0,1} \leq 0$. Then, we have

$$
\begin{aligned}
D_{1, \underline{c}_{1}+1} & =p_{2^{m}-N-1}+p_{2^{m}-N}-p_{1} \geq 2 p_{2^{m}-N+1}-p_{1} \\
& \geq 2\left(p_{N-1}+p_{N}\right)-p_{1} \geq 4 p_{N}-p_{1} \geq 0,
\end{aligned}
$$

where the equality follows from $(10)$ and the definition of $\underline{c}_{1}$, the first and third inequalities from the monotonicity of $\mathbf{p}$, the second inequality from our assumption on $D_{0,1}$, and the last inequality from the 4-uniformity of $\mathbf{p}$. Hence, we must have $D_{1, \underline{c}_{1}+1} \geq 0$. Similarly, if $D_{0,1}<0$, then we must have $D_{1, \underline{c}_{1}+1}>0$. Therefore, $-\operatorname{sg}\left(D_{1, \underline{c}_{1}+1}\right) \leq \operatorname{sg}\left(D_{0,1}\right)$, as claimed.

Proof of Theorem 3. The theorem follows directly from Lemma 8, observing also that by the assumptions of the theorem, and by Lemma 7, at least one of the trees $T_{\sigma, c}$, $\left(1, \bar{c}_{1}\right) \preceq(\sigma, c) \preceq\left(0, \bar{c}_{0}\right)$ must be optimal for $\mathbf{p}$.

\section{APPENDIX B \\ PROOFS FOR SUBSECTION IV-C}

We derive the proof of Theorem 4 through a series of lemmas. We recall that we seek an optimal tree for the source 
$\hat{\mathcal{A}}_{k}$ of $(7)$, with vector of (unnormalized) weights

$$
\mathbf{p}=\left(q^{0}, q^{1}, q^{1}, \ldots, q^{j}, q^{j}, \ldots, q^{j}, \ldots, q^{2 k-3}, q^{2 k-3}, q^{2 k-2}\right),
$$

with $q=2^{-1 / k}$, and where $q^{j}$ is repeated $j+1$ times for $0 \leq j \leq k-1$, and $2 k-1-j$ times for $k \leq j \leq 2 k-2$. For succinctness, in this appendix, when we say "optimal" we mean "optimal for $\hat{\mathcal{A}}_{k}$." Notice that, in $\mathbf{p}$, three consecutive weights are never distinct; we refer to this fact as the "three consecutive weights" property. Throughout the appendix, we assume that $k>2$, as we recall that optimal trees for $k=1,2$ are fully characterized in Remark 2 following Theorem 2

Lemma 12: Trees $T_{\sigma, c}$ with $c=\bar{c}_{\sigma}$ are not optimal. Consequently, the profile $\left(n_{M-1}, n_{M}, n_{M+1}\right)$ of an optimal tree has $n_{M} \geq 3$.

Proof: Recalling the profile $\mathbf{N}_{T_{\sigma, c}}$ in 8 , with $c=\bar{c}_{\sigma}$ and $k>2$, we have $n_{M} \in\{0,1,2\}, n_{M-1} \geq 1$ and $n_{M+1} \geq 2$. Let $q^{\ell}$ be the lightest weight on level $M-1$. By the "three consecutive weights" property, the two heaviest weights on level $M+1$ are greater than or equal to $q^{\ell+2}$. Recalling the expression for $D_{\sigma, c}$ in 10 , and the interpretation that follows it, we obtain $D_{\sigma, \bar{c}_{\sigma}} \geq q^{\ell}\left(1-2 q^{2}\right)>0$. Thus, by Theorem 3 . $T_{\bar{c}_{\sigma}}$ is not optimal. An optimal tree $T_{\sigma, c}$ would, therefore, have $c<\bar{c}_{\sigma}$, and, thus, $n_{M} \geq 3$.

The following lemma gives a first, rough approximation of the distribution of weights by levels in an optimal tree $T_{\sigma, c}$, which will allow us to identify the appropriate range (i.e., 12 or (13) for the heaviest and the lightest weights on level $M$ of the tree.

Lemma 13: Let $T_{\sigma, c}$ be an optimal tree, and let $q^{j}$ and $q^{2 k-2-j^{\prime}}$ denote, respectively, the heaviest and the lightest weights on level $M$ of the tree. Then, we have $j \leq k-1$, $j^{\prime} \leq k-1$, and $j+j^{\prime} \leq k$.

Proof: Consider first the case where $c>\underline{c}_{\sigma}$, i.e., all the components of the profile $\mathbf{N}_{T_{\sigma, c}}$ are positive. The lightest weight on level $M-1$ of the tree immediately precedes $q^{j}$ in p. Hence, it is of the form $q^{j-\varepsilon}$, with $\varepsilon \in\{0,1\}$. On the other hand, reasoning similarly, the heaviest two weights on level $M+1$ are of the form $q^{2 k-2-j^{\prime}+\varepsilon^{\prime}}$ and $q^{2 k-2-j^{\prime}+\varepsilon^{\prime}+\varepsilon^{\prime \prime}}$, where $\varepsilon^{\prime}, \varepsilon^{\prime \prime} \in\{0,1\}$ and $\varepsilon^{\prime}+\varepsilon^{\prime \prime} \leq 1$ (due to the "three consecutive weights" property). Since $T_{\sigma, c}$ is optimal, by the definition of $D_{\sigma, c}$ in (9), we must have $D_{\sigma, c} \leq 0$. Applying (10), the above constraints on $\varepsilon, \varepsilon^{\prime}, \varepsilon^{\prime \prime}$, and the fact that $q^{k}=\frac{1}{2}$, we get

$$
\begin{aligned}
0 \geq D_{\sigma, c} & =-q^{j-\epsilon}+q^{2 k-2-j^{\prime}+\epsilon^{\prime}}+q^{2 k-2-j^{\prime}+\epsilon^{\prime}+\epsilon^{\prime \prime}} \\
& \geq-q^{j-1}+2 q^{2 k-1-j^{\prime}}=-q^{j-1}+q^{k-1-j^{\prime}} .
\end{aligned}
$$

Thus, $j+j^{\prime} \leq k$. Since both $j$ and $j^{\prime}$ are positive when $c>\underline{c}_{\sigma}$, the claim of the lemma follows in this case.

Consider now the case where $c=\underline{c}_{\sigma}$, i.e., $T_{\sigma, c}$ is a quasiuniform tree. If $\sigma=0$, we have $n_{M+1}=0$, and, thus, the lightest weight on level $M$ is $p_{k^{2}}=q^{2 k-2}$, and $j^{\prime}=0$. For the heaviest weight on level $M$, we have $p_{2^{m}-k^{2}+1}=q^{j}$. By (14), we have $2^{m}-k^{2}+1 \leq k(k+1) / 2$. Recalling the order and structure of $\mathbf{p}$, we obtain $q^{j}=p_{2^{m}-k^{2}+1} \geq p_{k(k+1) / 2}=$ $q^{k-1}$. Thus, $j \leq k-1$. The case of $c=\underline{c}_{\sigma}$ and $\sigma=1$ is argued similarly, using (16) in lieu of (14), and leading to $j=0$ and $j^{\prime} \leq k-1$.
It follows from Lemma 13 that in an optimal tree, the heaviest weight on level $M$ is covered by (12) in Lemma 9 (and, thus, so is any weight on level $M-1$ ), while the lightest weight on level $M$ is covered by (13) in that lemma (and, thus, so is any weight on level $M+1)$. Consequently, an optimal tree is completely determined by a tuple $\mathbf{j}=\left(j, r, j^{\prime}, r^{\prime}\right)$, with $0 \leq j, j^{\prime} \leq k-1,0 \leq r \leq j$, and $0 \leq r^{\prime} \leq j^{\prime}$. The profile of the tree is then given by

$$
\begin{aligned}
n_{M-1} & =\frac{j(j+1)}{2}+r, \\
n_{M+1} & =\frac{j^{\prime}\left(j^{\prime}+1\right)}{2}+r^{\prime}, \\
n_{M} & =k^{2}-n_{M-1}-n_{M+1} .
\end{aligned}
$$

The following lemma presents a characterization of the least value of $c$ for which $T_{\sigma, c}$ is optimal. The lemma follows immediately from Theorem 3 and Lemma 10

Lemma 14: Let $c_{k}$ be the least value of $c$ such that $T_{\sigma, c}$ is optimal. Then, either $D_{\sigma, \underline{c}_{\sigma}+1} \geq 0$ (with $c_{k}=\underline{c}_{\sigma}$ ), or $D_{\sigma, c_{k}}<0$ and $D_{\sigma, c_{k}+1} \geq 0$ (with $c_{k}>\underline{c}_{\sigma}$ ).

Define the function

$F\left(j, r, j^{\prime}, r^{\prime}\right)=2 k^{2}-2^{M+1}+j(j+1)+2 r-\frac{j^{\prime}\left(j^{\prime}+1\right)}{2}-r^{\prime}$,

acting on tuples $\mathbf{j}=\left(j, r, j^{\prime}, r^{\prime}\right)$ for a given value of $k$. Next, we derive a set of conditions on the tuple $\mathbf{j}$ corresponding to the tree $T_{\sigma, c_{k}}$ characterized in Lemma 14

Lemma 15: Let $\mathbf{j}=\left(j, r, j^{\prime}, r^{\prime}\right)$ be the tuple defining the profile of $T_{\sigma, c_{k}}$ in (40)-(42). Then,

$$
F\left(j, r, j^{\prime}, r^{\prime}\right)=0
$$

and exactly one of the following conditions holds:

(i) $j, j^{\prime}>0, j+j^{\prime}=k-2$. Either $r=0$ and $0 \leq r^{\prime} \leq j^{\prime}$, or $1 \leq r \leq j$ and $r^{\prime} \in\{0,1\}$.

(ii) $j, j^{\prime}>0, j+j^{\prime}=k-1, r=0$ and $r^{\prime} \in\{0,1\}$.

(iii) $j^{\prime}=0, r^{\prime}=0, j \in\{k-2, k-1\}, 0 \leq r \leq j$.

(iv) $j=0, r=0, j^{\prime} \in\{k-2, k-1\}, 0 \leq r^{\prime} \leq j^{\prime}$.

Conversely, if $\mathbf{j}=\left(j, r, j^{\prime}, r^{\prime}\right)$ satisfies (44) and one of the conditions (i)-(iv), then $\mathbf{j}$ defines $T_{\sigma, c_{k}}$.

Proof: The necessity of (44) follows from the definition of $F\left(j, r, j^{\prime}, r^{\prime}\right)$ and from 38 , setting $c=\frac{1}{2} n_{M+1}$, substituting the expressions from (40) and (41) for $n_{M-1}$ and $n_{M+1}$, respectively, and rearranging terms. In fact, (44) must hold for any optimal tree, not just for $c=c_{k}$. Conditions (i)-(iv) will follow from an exhaustive case study of configurations that yield the inequalities on the quantities $D_{\sigma, c}$ that characterize the point $c=c_{k}$, as stated in Lemma 14

Consider, first, the case where $c_{k}>\underline{c}_{\sigma}$. Then, for $c=c_{k}$, by Lemma 14, we have $D_{\sigma, c}<0$ and $D_{\sigma, c+1} \geq 0$. Writing down the expressions for $D_{\sigma, c}$ and $D_{\sigma, c+1}$ explicitly according to 10 , we observe that six weights are involved, as illustrated in Figure 11. In order to switch from a negative $D_{\sigma, c}$ to a nonnegative $D_{\sigma, c+1}$, we must have a decrease from $p_{2^{M}-k^{2}+c}$ to $p_{2^{M}-k^{2}+c+1}$, or an increase from $p_{k^{2}-2 c+1}+p_{k^{2}-2 c+2}$ to $p_{k^{2}-2 c-1}+p_{k^{2}-2 c}$, or both. By the definitions of $j$ and $j^{\prime}$, we have $p_{2^{M}-k^{2}+c+1}=q^{j}$, and $p_{k^{2}-2 c}=q^{2 k-2-j^{\prime}}$. Taking into 


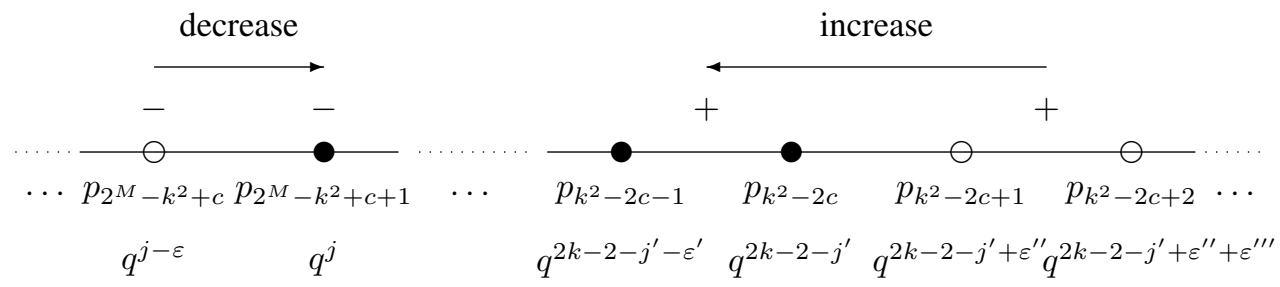

Fig. 11. Weights involved in the conditions for $c=c_{k}$ : $\bigcirc$ weights in $D_{\sigma, c}$, weights in $D_{\sigma, c+1}$.

account that consecutive weights can vary at most by a factor of $q$, we can write, for the other weights involved,

$$
\begin{aligned}
p_{2^{M}-k^{2}+c} & =q^{j-\varepsilon}, \\
p_{k^{2}-2 c-1} & =q^{2 k-2-j^{\prime}-\varepsilon^{\prime}}, \\
p_{k^{2}-2 c+1} & =q^{2 k-2-j^{\prime}+\varepsilon^{\prime \prime}}, \\
p_{k^{2}-2 c+2} & =q^{2 k-2-j^{\prime}+\varepsilon^{\prime \prime}+\varepsilon^{\prime \prime \prime}},
\end{aligned}
$$

where $\varepsilon, \varepsilon^{\prime}, \varepsilon^{\prime \prime}, \varepsilon^{\prime \prime \prime} \in\{0,1\}$, and, due to the "three consecutive weights" property, we must have $\varepsilon^{\prime}+\varepsilon^{\prime \prime} \leq 1$ and $\varepsilon^{\prime \prime}+\varepsilon^{\prime \prime \prime} \leq 1$. Table $\mathrm{V}$ ] summarizes the patterns of values of $\boldsymbol{\varepsilon}=\left(\varepsilon, \varepsilon^{\prime}, \varepsilon^{\prime \prime}, \varepsilon^{\prime \prime \prime}\right)$ that satisfy these constraints and also produce the combination of weight increases or decreases necessary to satisfy the conditions for $c=c_{k}$. On the right column of the table, we list the conditions imposed on $\mathbf{j}$ by the constraints of each case. To illustrate the proof approach, we derive these conditions, below, for the representative case $\boldsymbol{\varepsilon}=(1,0,0,1)$. The other cases follow using similar arguments, which are also similar to those used in the proof of Lemma 13 (here, more parameters are assumed known, which allows us to obtain tighter bounds).

Assume $\boldsymbol{\varepsilon}=(1,0,0,1)$. Then, writing the conditions on $D_{\sigma, c}$ and $D_{\sigma, c+1}$ at $c=c_{k}$ explicitly, substituting for the weights using the known values in $\boldsymbol{\varepsilon}$, and recalling that $q^{k}=\frac{1}{2}$, we obtain

$$
\begin{aligned}
0 & >D_{\sigma, c}=p_{k^{2}-2 c+1}+p_{k^{2}-2 c+2}-p_{2^{M}-k^{2}+c} \\
& =q^{2 k-2-j^{\prime}}+q^{2 k-1-j^{\prime}}-q^{j-1} \\
& >2 q^{2 k-1-j^{\prime}}-q^{j-1}=q^{k-1-j^{\prime}}-q^{j-1}
\end{aligned}
$$

and

$$
\begin{aligned}
0 & \leq D_{\sigma, c+1}=p_{k^{2}-2 c-1}+p_{k^{2}-2 c}-p_{2^{M}-k^{2}+c+1} \\
& =q^{2 k-2-j^{\prime}}+q^{2 k-2-j^{\prime}}-q^{j} \\
& =2 q^{2 k-2-j^{\prime}}-q^{j}=q^{k-2-j^{\prime}}-q^{j}
\end{aligned}
$$

It follows that $k-2 \leq j+j^{\prime} \leq k-1$, as claimed in the second row of Table $\overline{\mathrm{V}}$ The conditions on $r$ and $r^{\prime}$ follow from Lemma 9, observing that $r$ resets to zero at points where $j$ increases, and similarly with $r^{\prime}$ relative to $j^{\prime}$. In this case, $p_{2^{M}-k^{2}+c}$ is the last weight of the form $q^{j-1}$, and, thus, we have $n_{M-1}=2^{M}-k^{2}+c=j(j+1) / 2$ and $r=0$; scanning $\mathbf{p}$ from right to left, $p_{k^{2}-2 c+2}$ is the last weight of the form $q^{2 k-1-j^{\prime}}$, and, thus, we have $n_{M+1}=2 c=j^{\prime}\left(j^{\prime}+1\right) / 2+1$, and $r^{\prime}=1$.

It is readily verified that all the cases on the right column of Table $\mathrm{V}$ satisfy either Condition (i) or Condition (ii) of the lemma.
TABLE V

THE POSSIBLE CASES FOR $\boldsymbol{\varepsilon}=\left(\varepsilon, \varepsilon^{\prime}, \varepsilon^{\prime \prime}, \varepsilon^{\prime \prime \prime}\right)$ FROM 45 - 48, AND THE CONDITIONS IMPOSED ON $\left(j, r, j^{\prime}, r^{\prime}\right)$ AT $c=c_{k}$.

\begin{tabular}{|l|l|}
\hline$\left(\varepsilon, \varepsilon^{\prime}, \varepsilon^{\prime \prime}, \varepsilon^{\prime \prime \prime}\right)$ & Conditions on $\left(j, r, j^{\prime}, r^{\prime}\right)$ \\
\hline$(1,0,0,0)$ & $j+j^{\prime}=k-2, r=0,2 \leq r^{\prime} \leq j^{\prime}-1$ \\
\hline$(1,0,0,1)$ & $j+j^{\prime} \in\{k-2, k-1\}, r=0, r^{\prime}=1$ \\
\hline$(1,0,1,0)$ & $j+j^{\prime} \in\{k-2, k-1\}, r=0, r^{\prime}=0$ \\
\hline$(0,0,0,1)$ & $j+j^{\prime}=k-2,1 \leq r \leq j, r^{\prime}=1$ \\
\hline$(0,0,1,0)$ & $j+j^{\prime}=k-2,1 \leq r \leq j, r^{\prime}=0$ \\
\hline$(1,1,0,0)$ & $j+j^{\prime}=k-2, r=0, r^{\prime}=j^{\prime}$ \\
\hline$(1,1,0,1)$ & $j+j^{\prime} \in\{k-1, k-2\}, r=0, r^{\prime}=j^{\prime}=1$ \\
\hline$(0,1,0,0)$ & case cannot occur at $c=c_{k}$ \\
\hline$(0,1,0,1)$ & $j+j^{\prime}=k-2,1 \leq r \leq j, r^{\prime}=j^{\prime}=1$ \\
\hline
\end{tabular}

Consider now the case where $c_{k}=\underline{c}_{\sigma}$. In this case, the tree is quasi-uniform. When $\sigma_{k}=0$, since $n_{M+1}=0$, we have $j^{\prime}=r^{\prime}=0$. The condition $j \leq k-1$ was established in Lemma 13, while the condition $j \geq k-2$ follows directly from $D_{\sigma, \underline{c}_{\sigma}+1}=D_{\sigma, 1} \geq 0$. Thus, Condition (iii) of the lemma is satisfied in this case. Similarly, when $c_{k}=\underline{c}_{\sigma}$ and $\sigma_{k}=1$, we have $j=r=0, j^{\prime} \leq k-1$ was established in Lemma 13 and $j^{\prime} \geq k-2$ follows from $D_{\sigma, \underline{c}_{\sigma}} \geq 0$. Thus, Condition (iv) of the lemma is satisfied in this case.

To prove the sufficiency of the conditions of the lemma, we first claim that, with $\mathbf{j}$ satisfying the conditions, the profile $\mathbf{N}=\left(n_{M-1}, n_{M}, n_{M+1}\right)$ defined in 40-42) defines a valid tree. Clearly, $n_{M-1}$ and $n_{M+1}$ are non-negative. To verify that $n_{M}$ is also non-negative, we write

$$
\begin{aligned}
n_{M-1}+n_{M+1} & =\frac{j(j+1)}{2}+\frac{j^{\prime}\left(j^{\prime}+1\right)}{2}+r+r^{\prime} \\
& <\frac{\left(j+j^{\prime}+1\right)^{2}}{2}+j+j^{\prime}
\end{aligned}
$$

where the inequality follows from the fact that $(a+b+1)^{2}>$ $a(a+1)+b(b+1)$ for $a, b \geq 0$, and from the inequalities $r \leq$ $j$ and $r^{\prime} \leq j^{\prime}$. With $j+j^{\prime} \leq k-1$, it follows that $n_{M-1}+$ $n_{M+1}<k-1+k^{2} / 2<k^{2}$. Hence, $n_{M}$, as defined in (42), is positive. On the other hand, (44), together with the fact that the components of $\mathbf{N}$ add up to $k^{2}$, is equivalent to the Kraft equality for $\mathbf{N}$. Therefore, $\mathbf{N}$ defines a valid tree $T_{\sigma, c}$. It is readily verified that if either Condition (i) or (ii) is satisfied, then the parameters $(\sigma, c)$ of $T_{\sigma, c}$ satisfy $c>\underline{c}_{\sigma}, D_{\sigma, c}<$ 0 , and $D_{\sigma, c+1} \leq 0$. Thus, by Lemma 8, we have $c=c_{k}$. Similarly, if either Condition (iii) or (iv) is satisfied, we have $c=\underline{c}_{\sigma}, D_{\sigma, \underline{c}_{\sigma}+1} \geq 0$, and, again, $c=c_{k}$.

The following lemma explores some properties of the function $\Delta(x)$ defined in 17 . 
Lemma 16: (i) For any $x$, we have $\Delta(x+1)=\Delta(x)+x+k$.

(ii) We have $\Delta(-1) \leq 0$ and $\Delta(k)>0$. Thus, $x_{0}$, the largest real root of $\Delta$, satisfies $-1 \leq x_{0}<k$.

(iii) The values $\Delta(k-1)$ and $\Delta(k-2)$ are even integers.

Proof: (i) The claim is readily verified by direct application of (17).

(ii) Setting $x=-1$ in (17), and recalling that $Q=k^{2}-$ $\lceil k(k-1) / 4\rceil$ and $M=\lceil\log Q\rceil$, we obtain

$$
\begin{aligned}
\Delta(-1) & =2\left(k^{2}-\frac{k(k-1)}{4}-2^{M}\right) \\
& =2\left(Q-2^{M}+\frac{1}{2} \mathbf{1}_{(k \bmod 4) \in\{2,3\}}\right) \\
& =\mathbf{1}_{(k \bmod 4) \in\{2,3\}}+2\left(Q-2^{M}\right),
\end{aligned}
$$

where $\mathbf{1}_{\mathcal{P}}=1$ if the predicate $\mathcal{P}$ is true, or $\mathbf{1}_{\mathcal{P}}=0$ otherwise. It follows that $\Delta(-1)$ can be positive only if $(k \bmod 4) \in\{2,3\}$ and $Q=2^{M}$. Writing $Q=Q(k)$, and computing explicitly $Q(4 \ell+2)=(4 \ell+3)(3 \ell+1)$ and $Q(4 \ell+3)=(\ell+1)(12 \ell+7)$, we conclude that $Q$ has at least one odd divisor when $(k \bmod 4) \in\{2,3\}$. Therefore, we must have $\Delta(-1) \leq 0$.

Furthermore, since $Q \leq 2^{M} \leq 2 Q-1$, we have

$$
\begin{aligned}
\Delta(k) & =2 k^{2}-2^{M+1}+k(k+1)-1 \\
& \geq 2 k^{2}-4 Q+k(k+1)+1 \\
& =-2 k^{2}+4\left\lceil\frac{k(k-1)}{4}\right\rceil+k(k+1)+1 \\
& \geq-2 k^{2}+k(k-1)+k(k+1)+1=1 .
\end{aligned}
$$

Thus, $\Delta(k)>0$, and, since the coefficient of $x^{2}$ in $\Delta(x)$ is $\frac{1}{2}, x_{0}$ must be in the claimed range.

(iii) By direct computation, we have $\Delta(k-1)=2 k^{2}-$ $2^{M+1}+(k-1) k$ and $\Delta(k-2)=2 k^{2}-2^{M+1}+(k-2)(k-1)$. Since $k>2$ and $M>0$, both values are even.

To complete the proof of Theorem 4, we will construct a tuple $\mathbf{j}=\left(j, r, j^{\prime}, r^{\prime}\right)$ that satisfies the conditions of Lemma 15 . and, thus, defines the sought parameter pair $\left(\sigma_{k}, c_{k}\right)$.

Proof of Theorem 4. It follows immediately from the definition of $\Delta(x)$ in 177 and of $F\left(j, r, j^{\prime}, r^{\prime}\right)$ in 43 that for $j, r, j^{\prime}, r^{\prime}$ we have

$$
\begin{aligned}
& F\left(j, r, j^{\prime}, r^{\prime}\right) \\
& \quad=\Delta(j)+\frac{(k-j-2)(k-j-1)}{2}-\frac{j^{\prime}\left(j^{\prime}+1\right)}{2}+2 r-r^{\prime} .
\end{aligned}
$$

When $j^{\prime}=k-j-2$, this reduces to

$$
F\left(j, r, j^{\prime}, r^{\prime}\right)=\Delta(j)+2 r-r^{\prime},
$$

while with $j^{\prime}=k-1-j$ we get

$$
F\left(j, r, j^{\prime}, r^{\prime}\right)=\Delta(j)+2 r-r^{\prime}-(k-j-1) .
$$

We will use these relations to verify that the solutions constructed below satisfy (44). Let $x_{0}$ be the largest real root of $\Delta(x)$, and let $\xi=\left\lfloor x_{0}\right\rfloor$. By Lemma 16 (ii), we have $-1 \leq \xi<k, \Delta(\xi) \leq 0$, and $\Delta(\xi+1)>0$. We consider three main cases for $\Delta(\xi)$, and for each case (and possible sub-cases) we define a tuple $\mathbf{j}=\left(j, r, j^{\prime}, r^{\prime}\right)$ and verify that it satisfies the conditions of Lemma 15
1) $0 \leq-\Delta(\xi) \leq 2 \xi$ : Let $j=\xi, r=\left\lfloor\frac{-\Delta(j)+1}{2}\right\rfloor$ and $r^{\prime}=-\Delta(j) \bmod 2$. By the assumptions of the case on $\Delta(\xi)$, we have $j \geq 0$. As for $j^{\prime}$, we have the sub-cases below. At the end of each sub-case, we note which of Conditions (i)-(iv) of Lemma 15 is satisfied.

a) $j=0$ : We must have $\Delta(0)=0$, so we get $r=$ $r^{\prime}=0$, and we set $j^{\prime}=k-2$ (Condition (iv)).

b) $j \in\{k-2, k-1\}$ : By Lemma 16 (iii), $\Delta(j)$ is even, and $r^{\prime}=0$. We get $r=-\frac{\Delta(j)}{2}$ and $0 \leq r \leq j$ by the assumptions on $\Delta(\xi)$, and we set $j^{\prime}=0$ (Condition (iii)).

c) $0<j<k-2$ : Set $j^{\prime}=k-2-j$. From the choices for $r$ and $r^{\prime}$, we get $0 \leq r \leq j$ and $0 \leq r^{\prime} \leq 1 \leq j^{\prime}$ (Condition (i)).

To verify that (44) is satisfied, we apply (49) for subcases a) and c), and for sub-case b) with $j=k-2$. We apply (50) for sub-case b) with $j=k-1$. For example, for sub-case c), by (49) and the definitions of $r$ and $r^{\prime}$, we have,

$$
\begin{aligned}
F\left(j, r, j^{\prime}, r^{\prime}\right) & =\Delta(j)+2 r-r^{\prime} \\
& =\Delta(j)+2\left\lfloor\frac{1-\Delta(j)}{2}\right\rfloor-r^{\prime} \\
& =\Delta(j)+2 \frac{r^{\prime}-\Delta(j)}{2}-r^{\prime}=0 .
\end{aligned}
$$

Verification of $F=0$ for the other sub-cases follows along similar lines.

2) $-\Delta(\xi) \in\{2 \xi+1,2 \xi+2\}$ : Let $j=\xi+1$. By Lemma 16(ii), we have $0 \leq j \leq k$. We claim that $j \leq k-1$. Assume, contrary to the claim, that $j=k$. Then, $-\Delta(k-1)=-\Delta(\xi)=2 k-\varepsilon$ with $\varepsilon \in\{0,1\}$, and, by Lemma 16 (i), we have $\Delta(\xi+1)=\Delta(k)=$ $\Delta(k-1)+2 k-1=\varepsilon-1 \leq 0$, contradicting Lemma 16(ii), which establishes $\Delta(\xi+1)>0$. Thus, we have $0 \leq j \leq k-1$, and, defining $j^{\prime}=k-1-j$, we also have $0 \leq j^{\prime} \leq k-1$. By Lemma 16 (i), we have $\Delta(j)=\Delta(\xi+1)=\Delta(\xi)+\xi+k$, and, by the conditions of the case on $\Delta(\xi)$, we get $\Delta(j) \in\{k-j, k-j-1\}$. Define $r=0$, and $r^{\prime}=\Delta(j)-(k-j-1)$, which implies $r^{\prime} \in\{0,1\}$. Thus, whenever $0<j<k-1$, $\mathbf{j}=\left(j, r, j^{\prime}, r^{\prime}\right)$ satisfies Condition (ii) of Lemma 15 When $j=0$, $\mathbf{j}$ satisfies Condition (iv), and when $j=k-1$, it satisfies Condition (iii) as long as $r^{\prime}=0$. We claim that when $r^{\prime}=1$, we must have $j<k-1$. Otherwise, if $r^{\prime}=1$ and $j=k-1$, then, by the definition of $r^{\prime}$, we have $\Delta(k-1)=\Delta(j)=r^{\prime}+(k-j-1)=1$, contradicting Lemma 16 (iii). Thus, $\mathbf{j}$ satisfies one of the conditions (ii)-(iv) of Lemma 15 By (50) and the definitions of $r$ and $r^{\prime}, \mathbf{j}$ also satisfies (44).

3) $-\Delta(\xi) \geq 2 \xi+3$ : Let $j=\xi+1$. By Lemma 16(ii), we have $0 \leq j \leq k$. We claim that $j \leq k-2$. Assume, contrary to the claim, that $j=k-1$. Then, $\xi=k-2$, and, by the assumptions of the case, we have $-\Delta(k-$ 2) $\geq 2(k-2)+3=2 k-1$. Applying Lemma 16 (i), we get $\Delta(\xi+1)=\Delta(k-1)=\Delta(k-2)+(k-2)+k=$ $\Delta(k-2)+2 k-2 \leq-1$, contradicting Lemma 16(ii), since we must have $\Delta(\xi+1)>0$. Similarly, if $j=k$, 
then $-\Delta(k-1) \geq 2 k+1$ and $\Delta(k)=\Delta(k-1)+2 k-$ $1 \leq-2$, again contradicting Lemma 16 (ii). Thus, we have $0 \leq j \leq k-2$, and we can define $j^{\prime}=k-2-j$, which also satisfies $0 \leq j^{\prime} \leq k-2$. By Lemma 16(i), and the conditions of the case on $\Delta(\xi)$, we have $\Delta(j)=$ $\Delta(\xi+1)=\Delta(\xi)+\xi+k \leq k-\xi-3=k-2-$ $j=j^{\prime}$. Define $r=0$, and $r^{\prime}=\Delta(j)$, satisfying $0 \leq$ $r^{\prime} \leq j^{\prime}$. Thus, $\mathbf{j}=\left(j, r, j^{\prime}, r^{\prime}\right)$ satisfies Condition (i) of Lemma 15. By (49) and the definitions of $r$ and $r^{\prime}, \mathbf{j}$ also satisfies 44 .

Cases $1-3$ above cover all possible values of $\Delta(\xi)$, and in all cases, we have exhibited an explicit tuple $\mathbf{j}=\left(j, r, j^{\prime}, r^{\prime}\right)$ satisfying the conditions of Lemma 15 , and, therefore, defining the optimal tree $T_{\sigma_{k}, c_{k}}$. It can readily be verified that the definitions of $j$ and $r$ in (18) summarize the corresponding definitions in the cases of the proof, with the top branch of (18) corresponding to Case 11, and the bottom branch to Cases 2 and 3 Furthermore, the definition of $c_{k}$ in (19) reflects the parameter $c=n_{M-1}-2^{M}+k^{2}$ in the profile 40 -42 defined by $\mathbf{j}$ for $c=c_{k}$.

Proof of Corollary 17 By the structure of $C_{k}$ in Theorem 2, it suffices to prove that $Q_{k} \cdot Q_{k}$ is not optimal for the finite source $\mathcal{A}_{k}$. Let $h=\lceil\log k\rceil$ and $a=2^{h}-k$, with $0 \leq a<2^{h-1}$. From the profile of $Q_{k}$ given in in Section II-B. one derives the profile of $Q_{k} \cdot Q_{k}$, obtaining

$$
\mathbf{N}_{Q_{k} \cdot Q_{k}}=\left(n_{2 h-2}, n_{2 h-1}, n_{2 h}\right)=\left(a^{2}, 2 a(k-a),(k-a)^{2}\right) .
$$

Since $Q_{k} \cdot Q_{k}$ has fringe thickness $f_{T} \leq 2$, it has a representation $T_{\sigma_{g}}, c_{g}$, for some parameters $\sigma_{g}, c_{g}$, as defined in Lemma 7, with $N=k^{2}$. The case $a=0$ (i.e., $k=2^{h}$ ) is readily discarded as sub-optimal for $k>2$, as it corresponds to a uniform tree with $2^{2 h}$ leaves, which cannot be optimal for $\hat{\mathcal{A}}_{k}$ since $p_{k^{2}}+p_{k^{2}-1}<p_{1}$ for that source. Also, we can assume that $\sigma_{g}$ is such that Lemma 10 is satisfied, and that $n_{2 h-2}$ and $n_{2 h}$ are such that they can be written, respectively, as $n_{M-1}$ and $n_{M+1}$ in (40)-41, with $j$ and $j^{\prime}$ satisfying Lemma 13 Otherwise, $T_{\sigma_{g}}, c_{g}$ is not optimal, and the corollary is proved. By Lemma 9 , we can write $a^{2}<\frac{1}{2}(j+1)(j+2)<\frac{1}{2}(j+2)^{2}$, or $j>\sqrt{2 a-2 . ~ S i m i l a r l y, ~}$ we have $(k-a)^{2}<\frac{1}{2}\left(j^{\prime}+1\right)\left(j^{\prime}+2\right)<\frac{1}{2}\left(j^{\prime}+2\right)^{2}$, or $j^{\prime}>\sqrt{2}(k-a)-2$. Adding up, we obtain $j+j^{\prime}>\sqrt{2} k-4$, and, hence, for $k \geq 10, j+j^{\prime}>k$, contradicting Lemma 13 . For the remaining cases, if $k \in\{7,9\}$ one verifies that $\sigma_{g}$ violates Lemma 10 , and for $k \in\{3,5,6\}$, one can easily verify, by direct inspection, that $T_{\sigma_{g}, c_{g}}$ is sub-optimal for $\hat{\mathcal{A}}_{k}$.

\section{APPENDIX C \\ PROOFS FOR SUBSECTIONIV-D}

Proof of Corollary 2. By Theorem 2, the code length for $(a, b)$ under $C_{k}$ is $\left|T_{k}(a \bmod k, b \bmod k)\right|+2+\left\lfloor\frac{a}{k}\right\rfloor+\left\lfloor\frac{b}{k}\right\rfloor$. Writing $a=m k+i$ and $b=n k+j$ with $0 \leq i, j<k$, $m, n \geq 0$, the average code length under $C_{k}$ is

$$
\begin{aligned}
& \overline{\mathcal{L}}_{q}\left(C_{k}\right) \\
& =\left(1-q^{2}\right) \sum_{0 \leq i, j<k} \sum_{m, n \geq 0} q^{i+j+(m+n) k}\left(\left|T_{k}(i, j)\right|+m+n+2\right) \\
& =\frac{2}{1-q^{k}}+\frac{(1-q)^{2}}{\left(1-q^{k}\right)^{2}} \sum_{0 \leq i, j \leq k-1}\left|T_{k}(i, j)\right| q^{i+j} \\
& =\frac{2}{1-q^{k}}+\overline{\mathcal{L}}_{q}\left(T_{k}\right),
\end{aligned}
$$

where the second equality follows from elementary series computations, and the third identifies the (normalized) average code length of the code $T_{k}$ defined in Theorem 4 Denote by $W_{M-1}, W_{M}$, and $W_{M+1}$ the total normalized weight of symbols in $\hat{\mathcal{A}}_{k}$ assigned length $M-1, M$, and $M+1$, respectively, by $T_{k}$. Then, the average code length of $T_{k}$ is given by

$$
\begin{aligned}
\overline{\mathcal{L}}_{q}\left(T_{k}\right) & =(M-1) W_{M-1}+M W_{M}+(M+1) W_{M+1} \\
& =M+W_{M+1}-W_{M-1} .
\end{aligned}
$$

From the profile $(8)$, with $N=k^{2}$ and $c=c_{k}$ as defined in 19, recalling (12), letting $\gamma=(1-q)^{2} /\left(1-q^{k}\right)^{2}$, and carrying out the computations, we obtain

$$
\begin{aligned}
W_{M-1} & =\gamma \sum_{i=1}^{j(j+1) / 2+r} p_{i}=\gamma \sum_{\ell=0}^{j-1}(\ell+1) q^{\ell}+\gamma r q^{j} \\
& =\frac{1-q^{j}\left(1+(1-q) j-(1-q)^{2} r\right)}{\left(1-q^{k}\right)^{2}} .
\end{aligned}
$$

Similarly, from the proof of Theorem 4 setting $j^{\prime}=k-j-2$ and $r^{\prime}=2 r+\Delta(j)$, we obtain

$$
\begin{aligned}
& W_{M+1}=\gamma \sum_{i=0}^{j^{\prime}\left(j^{\prime}+1\right) / 2+r^{\prime}-1} p_{k^{2}-i} \\
& =\gamma \sum_{\ell=0}^{j^{\prime}-1}(\ell+1) q^{2 k-2-\ell}+\gamma r^{\prime} q^{2 k-2-j^{\prime}} \\
& =\frac{q^{2 k}+q^{k+j}\left((k-j-1)(1-q) q-q+(1-q)^{2}(2 r-\Delta(j))\right)}{\left(1-q^{k}\right)^{2}} .
\end{aligned}
$$

The result 20 now follows by substituting the above expressions for $W_{M-1}$ and $W_{M+1}$ in $\sqrt{52}$, substituting for $\overline{\mathcal{L}}_{q}\left(T_{k}\right)$ in (51), and using appropriate algebraic simplifications. The result $\left(21\right.$, in turn, follows by applying the relation $q^{k}=1 / 2$.

\section{APPENDIX D}

\section{LAYER TRANSITIONS IN THE CODES $C_{-k}$}

In each layer transition described below, we assume that we start from a layer $\mathbf{L}_{s}$ of type (x), and show how it unfolds into a layer $\mathbf{L}_{s+1}$ of type (y), the transition being denoted $(\mathrm{x}) \rightarrow(\mathrm{y})$. We denote by $d_{s}$ the depth of the shallowest node in $\mathbf{L}_{s}$.

(i) $\rightarrow$ (i): The tree $q^{s+1} \mathcal{V}_{k}$ in each of the $\ell$ groups $\mathcal{M}$ in $\mathbf{L}_{s}$ unfolds, by the definition of $\mathcal{V}_{k}$ (see also Figure 4), into a tree $q^{s+2} \mathcal{V}_{k}$ and $2^{k}-1$ leaves of weight $q^{s+1}$, which provides a group $\mathcal{M}$ for $\mathbf{L}_{s+1}$. Hence, there 
are $\ell$ groups $\mathcal{M}$ in $\mathbf{L}_{s+1}$, which include $\left(2^{k}-1\right) \ell$ signatures $s+1$. This propagation of groups $\mathcal{M}$ will occur in the same way in all the other transitions below; its discussion will be omitted for those cases. There remain $s+2-\left(2^{k}-1\right) \ell=2^{k-1}+1+j$ signatures $s+1$, with $0 \leq j \leq 2^{k-1}-4$ (recall that layers of type (i) exist only if $k>2$ ). A quasiuniform tree with $2^{k-1}+2+j$ leaves is built, rooted at $\mathcal{R}_{s}$. This tree has $2^{k-1}-(j+1)-1$ leaves at depth $k-1$, which are labeled $s+1$, and $2(j+1)+2$ leaves at depth $k$, of which $2(j+1)+1$ are assigned label $s+1$, and one serves as the root of $\mathcal{R}_{s+1}$, consistent with a structure of type (i) for $s+1$ (and, correspondingly, $j+1$ ).

(i) $\rightarrow$ (ii): We have $j=2^{k-1}-3$. We let $\mathcal{R}_{s}$ be the root of a balanced tree of height $k$. Of its $2^{k}$ leaves, $2^{k}-2$ are assigned the remaining $2^{k}-2$ signatures $s+1$, one leaf serves as the root for $q \mathcal{U}_{k-1}$, and the remaining leaf as the root for $\mathcal{R}_{s+1}$.

(ii) $\rightarrow$ (iii) $(k>2)$ : The tree $q \mathcal{U}_{k-1}$ in $\mathbf{L}_{s}$ contributes $2^{k-1}$ leaves of signature $s+1$ to $\mathbf{L}_{s+1}$, in addition to those contributed by the groups $\mathcal{M}$. There remain $2^{k-1}-1$ signatures $s+1$, which are assigned to leaves of a balanced tree $\mathcal{U}_{k-1}$ rooted at $\mathcal{R}_{s}$. The remaining leaf splits into two nodes, one is the root of a tree $q \mathcal{U}_{k-1}$, and the other anchors $\mathcal{R}_{s+1}$.

(ii) $\rightarrow$ (iv) $(k=2)$ : The tree $q \mathcal{U}_{1}$ in $\mathbf{L}_{s}$ contributes $2^{1}$ leaves of signature $s+1$ to $\mathbf{L}_{s+1}$, in addition to those contributed by the groups $\mathcal{M}$. The remaining signature $s+1$ is assigned to one leaf of a tree $\mathcal{U}_{1}$ rooted at $\mathcal{R}_{s}$. The second leaf splits into two nodes, one is the root of a tree $q \mathcal{V}_{k}^{-}$, and the other anchors $\mathcal{R}_{s+1}$.

(iii) $\rightarrow$ (iii): The construction from the previous transition is kept, except that one of the leaves of the tree $\mathcal{U}_{k-1}$ rooted at $\mathcal{R}_{s}$ is split, making room for the additional signature $s+1$ resulting from the increase in $s$. Hence, there is a decrease by one in the number of leaves at depth $d_{s}$ and an increase by two in the number of leaves at depth $d_{s}+1$. This process continues until $j=2^{k}-4$.

(iii) $\rightarrow$ (iv): This transition is identical to the previous one, except that instead of a tree $q \mathcal{U}_{k-1}$, a tree $q \mathcal{V}_{k}^{-}$is attached as sibling to $\mathcal{R}_{s+1}$.

(iv) $\rightarrow(\mathrm{v})$ : The tree $q \mathcal{V}_{k}^{-}$from the previous transition provides the $2^{k-1}-1$ leaves of signature $s+1$, plus a tree $q \mathcal{V}_{k}$. What started as a balanced tree of depth $k-1$ in the transition (ii) $\rightarrow$ (iii) has evolved into a balanced tree of depth $k$, with all leaves assigned signatures $s+1$, except for one, which serves as the root of $\mathcal{R}_{s+1}$.

(v) $\rightarrow$ (i) $(k>2)$ : The tree $q \mathcal{V}_{k}$ added in the previous transition generates a new group $\mathcal{M}$, consistent with the increment in $\ell$. All signatures $s+1$ now originate from the groups $\mathcal{M}$, or from $\mathcal{R}_{s}$, which brings the construction back to a layer of type (i), completing the cycle.

(v) $\rightarrow$ (ii) $(k=2)$ : When $k=2$ the transition occurs to a layer of type (ii), as described above for the initial transition from Case 1 to Case 2.

\section{REFERENCES}

[1] S. W. Golomb, "Run length encodings," IEEE Trans. Inf. Theory, vol. IT12, pp. 399-401, 1966.

[2] R. G. Gallager and D. C. Van Voorhis, "Optimal source codes for geometrically distributed integer alphabets," IEEE Trans. Inf. Theory, vol. IT-21, pp. 228-230, 1975.

[3] N. Merhav, G. Seroussi, and M. J. Weinberger, "Optimal prefix codes for sources with two-sided geometric distributions," IEEE Trans. Inf. Theory, vol. 46, pp. 229-236, 2000.

[4] R. F. Rice, "Some practical universal noiseless coding techniques," Tech. Rep. JPL-79-22, JPL, Pasadena, CA, 1979.

[5] M. J. Weinberger, G. Seroussi, and G. Sapiro, "The LOCO-I lossless image compression algorithm: Principles and standardization into JPEGLS," IEEE Trans. Image Proc., vol. 9, pp. 1309-1324, 2000.

[6] T. Linder, V. Tarokh, and K. Zeger, "Existence of optimal prefix codes for infinite source alphabets," IEEE Trans. Inf. Theory, vol. 43, pp. 20262028, 1997.

[7] A. Kato, T. S. Han, and H. Nagaoka, "Huffman coding with an infinite alphabet," IEEE Trans. Inf. Theory, vol. 42, pp. 977-984, 1996.

[8] J. Abrahams, "Code and parse trees for lossless source encoding," Commun. Inf. Syst., vol. 1, pp. 113-146, 2001.

[9] M. J. Golin and K. K. Ma, "Algorithms for constructing infinite Huffman codes,” Technical Report HKUST-TCSC-2004-07, HKUST, Hong Kong, China, July 2004.

[10] S. W. Golomb, "Sources which maximize the choice of a Huffman coding tree," Information and Control, vol. 45, pp. 263-272, jun 1980.

[11] M. B. Baer, Coding for General Penalties. PhD thesis, Stanford University, 2003.

[12] F. Bassino, M.-P. Béal, and D. Perrin, "A finite state version of the Kraft-McMillan theorem," SIAM Journal on Computing, vol. 30, no. 4, pp. 1211â-1230, 2000.

[13] T. M. Cover and J. A. Thomas, Elements of Information Theory. Wiley Series in Telecommunications and Signal Processing, WileyInterscience, 2 ed., 2006.

[14] M. Garey and D. S. Johnson, Computers and Intractability. New York: Freeman, 1979.

[15] G. Seroussi and M. J. Weinberger, "On adaptive strategies for an extended family of Golomb-type codes," in Proc. DCC'97, (Snowbird, UT), pp. 131-140, 1997. 